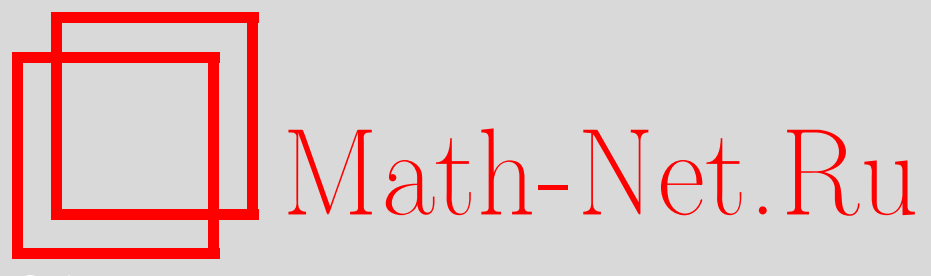

И. А. Богаевский, Разрывные градиентные дифференциальные уравнения и траектории в вариационном исчислении, Матем. сб., 2006, том 197, номер 12, 11-42

DOI: https://doi.org/10.4213/sm1502

Использование Общероссийского математического портала Math-Net.Ru подразумевает, что вы прочитали и согласны с пользовательским соглашением http: //www. mathnet.ru/rus/agreement

Параметры загрузки:

IP : 54.80 .97 .219

26 апреля 2023 г., $14: 45: 20$

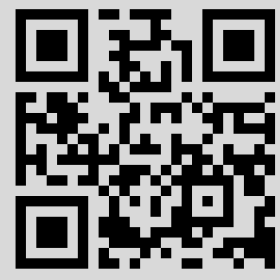




\title{
И. А. Богаевский
}

\section{Разрывные градиентные дифференциальные уравнения и траектории в вариационном исчислении}

\begin{abstract}
Понятие градиента гладких функций обобщается на их суммы с вогнутыми функциями. Для обыкновенного дифференциального уравнения, правая часть которого - градиент суммы вогнутой и гладкой функций, сформулирована и доказана теорема существования, единственности и непрерывной зависимости решения при возрастании времени. $\mathrm{C}$ ее помощью в вариационной задаче о наименьшем механическом действии в пространстве произвольной размерности строится физически естественное движение частиц, определенное даже на разрывах поля скоростей. При таком движении описаны все типичные случаи возникновения и взаимодействия на плоскости точечных скоплений частиц с положительной массой.
\end{abstract}

Библиография: 8 названий.

\section{Введение}

Теоремы существования и единственности решения, вообе говоря, не справедливы для обыкновенных дифференциальных уравнений с разрывной правой частью. Однако показанные на рис. 1 решения следующего дифференциального уравнения

$$
\dot{x}=-\operatorname{sign} x=\left\{\begin{array}{rc}
-1 & \text { при } x>0, \\
0 & \text { при } x=0, \\
1 & \text { при } x<0
\end{array}\right.
$$

все-таки удовлетворяют этим теоремам при возрастании времени. А именно, в каждой точке начинается одно и только одно решение, но заканчиваться может и больше. Кроме того, это решение непрерывно зависит от начальной точки. Иначе говоря, для нашего уравнения справедливы теоремы существования, единственности и непрерывной зависимости решения от начального условия при возрастании времени. Правда, показанные на рис. 1 кривые имеют изломы, поэтому требуется уточнить, в каком смысле они являются решениями нашего дифференциального уравнения. Ответ прост: вместо обычной производной по времени надо рассматривать одностороннюю справа, что тоже

Работа выполнена при поддержке Российского фонда фундаментальных исследований (грант № 05-01-00104), Программы поддержки ведущих научных школ РФ (грант № НШ4719.2006.1) и Министерства образования Франции.

(С) И. А. БогАевский, 2006 
согласуется с идеей возрастания времени - его приращение должно быть положительным. Иными словами, внешние условия, описываемые правой частью дифференциального уравнения, влияют на будущее, а не на прошлое.

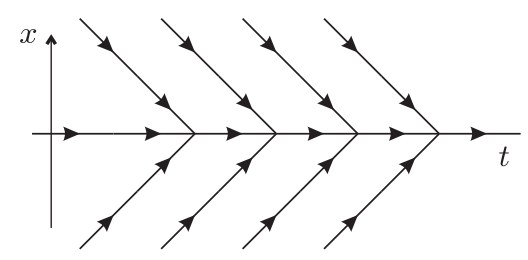

Рис. 1. Решения уравнения $\dot{x}=-\operatorname{sign} x$

В первой части настоящей статьи доказывается теорема 1, обобщающая приведенный выше пример. А именно, если правая часть обыкновенного дифференциального уравнения является градиентом некоторой функции, то оно называется градиентным, а сама функция - его потенциалом. Оказывается, что градиент можно определить не только для гладких ${ }^{1}$ функций, но и для вогнутых, а для градиентного дифференциального уравнения на евклидовом пространстве с вогнутым потенциалом справедливы теоремы о существовании, единственности и непрерывной зависимости решения от начального условия при возрастании времени. Более того, решение непрерывно зависит и от самого потенциала относительно топологии равномерной сходимости на компактах, называемой еще компактно-открытой.

Вогнутая функция - это функция с выпуклым подграфиком; известно, что она непрерывна и имеет производную вдоль любого направления, непрерывно зависящую от последнего. (Под направлением мы подразумеваем луч, а не прямую.) Этот факт позволяет однозначно определить градиент вогнутой функции стандартным образом - это вектор, указывающий направление ее максимального возрастания, длина которого равна ее производной в этом направлении; если же вогнутая функция во всех направлениях не возрастает, то ее градиент по определению равен нулю. Градиент определен единственным образом вследствие вогнутости исходной функции. Если же исходная вогнутая функция еще и гладкая, то получается ее обычный градиент. Однако для гладких функций градиент аддитивен (градиент суммы функций равен сумме их градиентов), а для вогнутых - вообще говоря, нет.

Приведенное выше дифференциальное уравнение - градиентное с вогнутым потенциалом $-|x|$. Приведем еще два примера из реальной жизни, демонстрирующих вышеприведенную теорему. У рек бывает много притоков, но, как правило, нет рукавов (раздвоений). Объяснение состоит в том, что обычно глубина долины реки является вогнутой функцией, градиент которой равен скорости течения реки. Второй пример предложил М.Э. Казарян: два альпиниста, взбирающихся из разных мест на выпуклую гору по градиенту ее высоты, после возможной встречи обязательно должны двигаться вместе.

\footnotetext{
1Термин "гладкий” здесь и далее означает “бесконечно дифференцируемый”.
} 
Основной же результат настоящей статьи - это теорема 2, обобщающая теорему 1 в следующих двух направлениях. Во-первых, можно ослабить требование вогнутости и потребовать от потенциала вогнутости лишь его разности с некоторой гладкой функцией. Такой потенциал мы будем называть (по предложению М.И. Зеликина) вогнутым относительно рассматриваемой гладкой функции или просто относительно вогнутым. (В частности, все гладкие и все вогнутые потенциалы являются относительно вогнутыми.) Определение градиента переносится дословно на относительно вогнутые потенциалы и для гладкого потенциала дает его обычный градиент. Если допустить уход решений в бесконечность за конечное время (т.е. отказаться от глобального существования решений), то теорема 1 остается справедливой для потенциалов, вогнутых относительно одной и той же гладкой функции.

Во-вторых, для физических приложений важно допустить зависимость потенциала от времени. Теорема 1 в этом случае становится неверной даже для потенциалов, вогнутых на пространстве-времени. Оказывается, надо еще модифицировать определение градиента. А именно, назовем полнъм градиентом относительно вогнутого потенциала $\varphi(x, t)$ в данной мировой точке $(x, t)$ скорость, при которой величина

$$
\frac{1}{2}|\dot{x}|^{2}-\dot{\varphi}
$$

достигает своего минимума, где второй член представляет собой полную производную потенциала, т.е. его производную вдоль мирового вектора $(\dot{x}, 1)$. Это и прежнее определение градиентов дают один и тот же результат для относительно вогнутых автономных потенциалов. Кроме того, полный градиент гладкого потенциала (даже зависящего от времени) совпадает с его обычным пространственным градиентом и полностью определяется поведением потенциала в данный момент времени. Однако полный градиент негладкого вогнутого потенциала, вообще говоря, зависит не только от его поведения в данный момент времени, но и от его последующего поведения.

Рассмотрим, например, вогнутый потенциал $\varphi(x, t)=-|x-w t|$ при различных значениях параметра $w \geqslant 0$. Его полная производная вычисляется по формуле

$$
\dot{\varphi}= \begin{cases}-\dot{x}+w & \text { при } x>w t, \\ -|\dot{x}-w| & \text { при } x=w t, \\ \dot{x}-w & \text { при } x<w t,\end{cases}
$$

и вышеприведенное условие минимальности дает

$$
\dot{x}= \begin{cases}-1 & \text { при } x>w t, \\ \min \{w, 1\} & \text { при } x=w t, \\ 1 & \text { при } x<w t .\end{cases}
$$

На рис. 2 изображены решения этого дифференциального уравнения при $0 \leqslant$ $w<1, w=1$ и $w>1$ соответственно. 

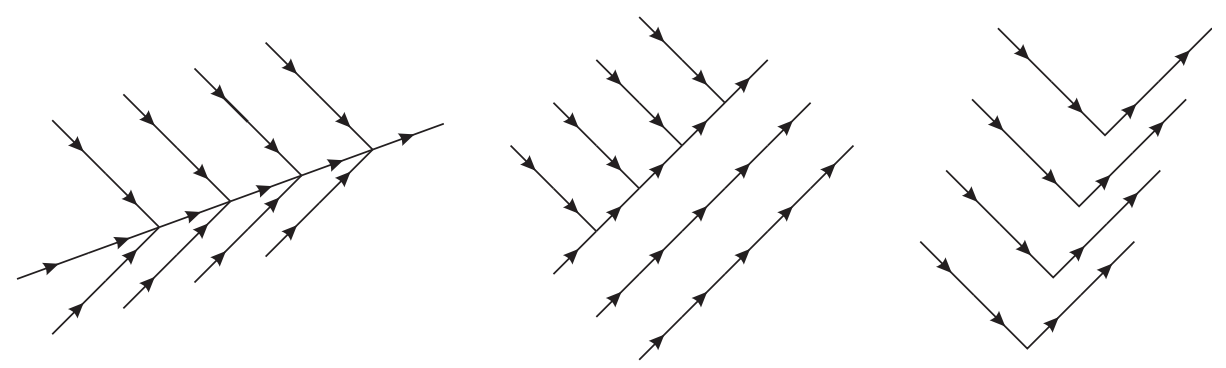

Рис. 2. Решения градиентного дифференциального уравнения с вогнутым потенциалом $-|x-w t|$ при $0 \leqslant w<1, w=1$ и $w>1$

Во второй части настоящей статьи теорема 2 применяется для построения траекторий частиц в вариационной задаче о наименьшем механическом (лагранжевом) действии с гладкими данными. Известно, что наименьшее действие является обобщенным решением соответствующего уравнения ГамильтонаЯкоби и равно пределу при исчезновении вязкости потенциала скоростей бесконечно сжимаемой вязкой жидкости, текущей во внешнем потенциальном силовом поле.

Даже если начальное распределение скоростей и внешнее силовое поле являются гладкими, у поля скоростей могут появляться разрывы, образующие ударную волну. В ее точках происходят столкновения частиц, т.е. заканчиваются по меньшей мере две оптимальные траектории, вдоль которых достигается минимум действия. Оказывается, теорема 2 позволяет корректно определить движение частиц внутри ударной волны. В частности, столкнувшиеся частицы слипаются и в дальнейшем двигаются как одно целое. Другими словами, между ними происходят абсолютно неупругие столкновения.

Процедура построения траекторий частиц такова. Наименьшее действие является (совсем необязательно гладкой) относительно вогнутой функцией, полный градиент которой, взятый в качестве скорости частицы, задает дифференциальное уравнение. Его решения естественно объявить траекториями частиц, поскольку согласно теореме 2 они однозначно определены и при исчезновении вязкости к ним сходятся траектории частиц, уже однозначно определенные ввиду гладкости поля скоростей при ненулевой вязкости. Оказывается, что построенное разрывное поле скоростей, задаваемое полным градиентом наименьшего действия, удовлетворяет следующему простому правилу, позволяющему эффективно его вычислять. А именно, скорость частицы совпадает с центром шара минимального радиуса, содержащего скорости всех оптимальных траекторий, заканчивающихся в точке, где находится частица в рассматриваемый момент времени. Иначе говоря, скорость частицы - это центр минимального шара, содержащего скорости всех частиц, бесконечно близких к данной в рассматриваемый момент времени.

Опишем более подробно, что происходит в двумерном случае. При типичных начальных условиях ударная волна является (вообе говоря, незамкнутой) 
гладкой кривой с некоторыми предписанными особенностями. В типичные моменты времени единственные особенности ударной волны - это тройные точки или узлы, пример такой ударной волны показан на рис. 3. Однако в отдельные моменты времени могут появляться новые особенности, а сама волна испытывает перестройки, которые при типичных начальных условиях исчерпываются показанными на рис. 4, заимствованному из [1]. На этом рисунке в первой и третьей строке изображен фрагмент ударной волны соответственно до и после перестройки, во второй - он же в сам момент перестройки. Рисунки типичных перестроек ударных волн в трехмерном пространстве можно найти также в [1].

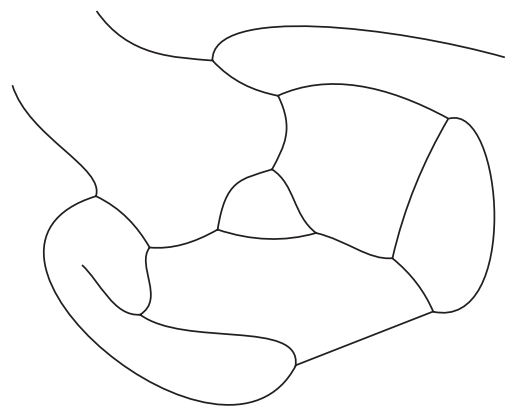

Рис. 3. Пример ударной волны на плоскости

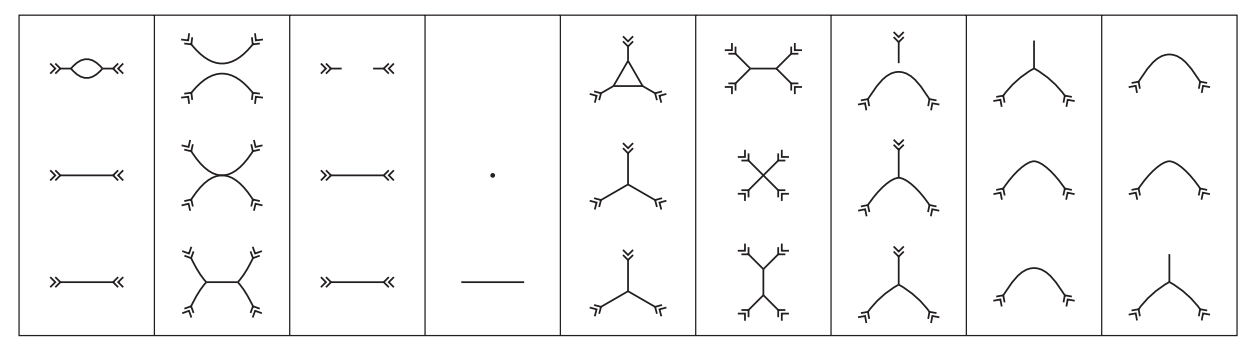

Рис. 4. Типичные перестройки ударной волны на плоскости

Естественно предположить, что линейная плотность частиц положительна на ударной волне. Это действительно так. Однако следующее естественное предположение о том, что узлы являются точечными скоплениями частиц с положительной массой или кластерами, оказывается неверным согласно нашим результатам. А именно, следует различать притягивающие (все три угла тупые) и нейтральные (один угол из трех острый) узлы. Согласно приведенным ниже вычислениям движение частиц в системе отсчета, связанной с узлом, в его окрестности устроено так, как показано на рис. 5. Из него видно, что растущие кластеры могут находиться лишь в притягивающих узлах, а через нейтральные частицы просто проходят без накапливания.

Опишем более подробно, как появляются и ведут себя кластеры с течением времени. Сначала ударной волны вообще нет, а потом после 4-й перестройки 

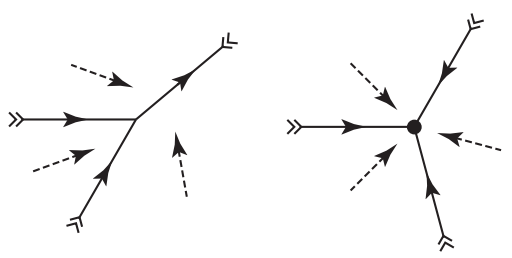

Рис. 5. Движение частиц около нейтрального и притягивающего узлов ударной волны

из рис. 4 она появляется, но пока на ней нет узлов. Возникновение узлов происходит во 2-й, 7-й и 9-й перестройках, но все эти узлы являются нейтральными, и накопления массы в них все еще не происходит. Более того, притягивающие узлы могут участвовать только в 5-й и 6-й перестройках, в течение которых узлы не появляются и не исчезают, а лишь взаимодействуют друг с другом.

Со временем нейтральный узел может стать притягивающим, после чего в нем начинается рост кластера. Кроме того, притягивающие узлы могут появляться в течение 5-й и 6-й перестроек, что также приводит к зарождению кластера. Все эти случаи показаны на рис. 6 .

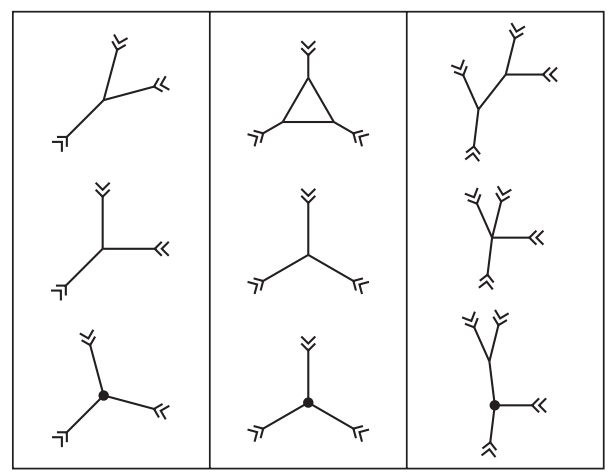

Рис. 6. Возникновение растущего кластера

Но со временем притягивающий узел может стать нейтральным, и находившийся в нем кластер останавливается в росте и начинает свободно двигаться вдоль ударной волны. То же самое может произойти и после 5-й и 6-й перестроек - см. рис. 7, на котором такие свободно дрейфующие стабильные кластеры показаны, в отличие от растущих, белыми кружками.

Два кластера могут столкнуться и образовать новый кластер, как это показано на рис. 8. Еще кластеры могут взаимодействовать с нейтральными узлами - на рис. 9 изображено, как это происходит. Однако согласно нашей теории ни раздваиваться, ни исчезать кластеры не могут.

В третьей части настоящей статьи мы обобщаем понятия градиента и полного градиента и включаем их в общую схему. А именно, мы рассматриваем про- 


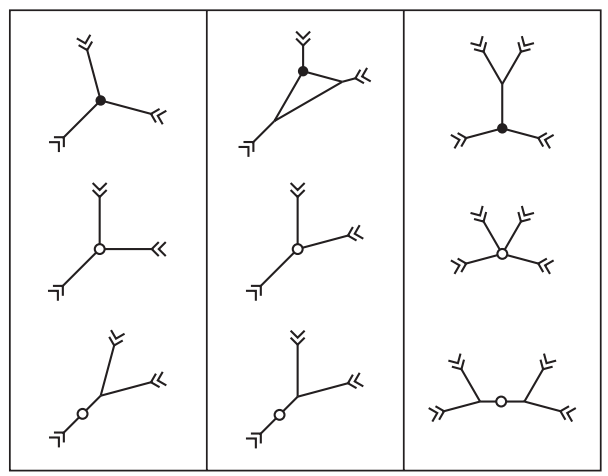

Рис. 7. Кластер перестает расти и покидает узел

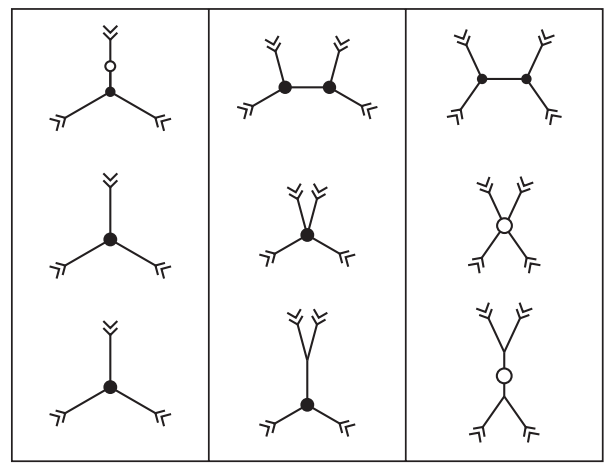

Рис. 8. Столкновение двух кластеров

извольное конечномерное вещественное пространство и фиксируем на его двойственном пространстве гладкую выпуклую функцию $h$, называемую в дальнейшем гамильтонианом. Далее для произвольной относительно вогнутой функции на исходном пространстве мы определяем ее $h$-градиент двумя эквивалентными способами - гамильтоновым и лагранжевым.

Например, если $h=|p|^{2} / 2$ - половина евклидова квадрата импульса, то получается градиент из первой части настоящей статьи. Полный градиент также укладывается в эту схему при $h=|p|^{2} / 2+q$, где $q$ - импульс, двойственный времени. Более точно, $h$-градиент в этом случае - это вектор в пространствевремени, временна́я координата которого равна единице, а пространственная компонента совпадает с полным градиентом.

В заключение мы формулируем естественную гипотезу, обобщающую локальный вариант теоремы 1 и теорему 2. А именно, для автономного $h$-градиентного дифференциального уравнения с относительно вогнутым потенциалом видимо справедливы теоремы о локальном существовании, единственности и непрерывной зависимости решения. Несмотря на подтверждающую ее теорему 3 о существовании решения для однородного вогнутого потенциала, эта 


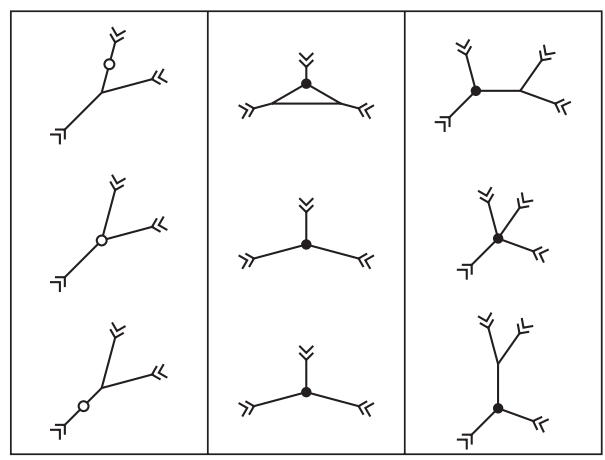

Рис. 9. Взаимодействие кластеров с нейтральными узлами

общая гипотеза остается недоказанной, поскольку для произвольного гамильтониана не удается доказать даже единственность решения.

Автор благодарит Д. В. Аносова, В. И. Арнольда, Ж. Бе́ка, А. В. Дмитрука, М. И. Зеликина, А. Н. Соболевского, А. М. Степина, У. Фриша и К. М. Ханина, чья помощь - в различной форме - была ему очень полезна.

\section{§ 1. Градиентные дифференциальные уравнения с вогнутыми потенциалами}

\section{1. Вогнутые функции, их дифференцируемость и сходимость.} Подмножество конечномерного вещественного линейного пространства $\Lambda$ называется выпуклым, если вместе со своими любыми двумя точками оно содержит соединяющий их отрезок. Вещественнозначная функция $f$, определенная на выпуклом подмножестве пространства $\Lambda$, называется вогнутой, если ее график лежит над всеми отрезками, соединяющими две любые его точки:

$$
f(\alpha x+\beta y) \geqslant \alpha f(x)+\beta f(y) \quad \forall \alpha, \beta>0, \quad \alpha+\beta=1 .
$$

Функция $f$ называется выпуклой, если противоположная к ней функция $-f$ является вогнутой. Вогнутость и выпуклость функций называются строгими, если указанное неравенство строгое. Хорошо известны (см., например, [2]) следующие свойства вогнутых функций.

Непрерывность [2; 10.1]. Вогнутая функция непрерывна во внутренних точках своей области определения.

Дифференцируемость [2; 23.1]. Вогнутая функция во внутренней точке своей области определения имеет конечную производную вдоль любого вектора:

$$
f_{x}^{\prime}(v)=\lim _{c \rightarrow+0} \frac{f(x+c v)-f(x)}{c} .
$$

Эта производная как функция от вектора $v$ вогнута, непрерывна и однородна степени 1:

$$
f_{x}^{\prime}(c v)=c f_{x}^{\prime}(v) \quad \forall c \geqslant 0
$$


(Непрерывность производной следует из ее вогнутости.)

Оценка через производную. Приращение вогнутой функции оценивается сверху ее производной:

$$
f_{x}^{\prime}(y-x) \geqslant f(y)-f(x) .
$$

Если же вогнутая функция является еще и однородной степени 1, то она сама оценивается своей производной в любой точке:

$$
f_{x}^{\prime}(v) \geqslant f(x+v)-f(x)=2 f\left(\frac{x}{2}+\frac{v}{2}\right)-f(x) \geqslant 2\left(\frac{f(x)}{2}+\frac{f(v)}{2}\right)-f(x)=f(v) .
$$

Сходимость $[2 ; 10.8]$. Поточечная сходимость вогнутых функций на открытом выпуклом множестве является равномерной на любом компакте, а в пределе всегда получается вогнутая функция.

1.2. Градиент вогнутой функции. Скалярное произведение векторов в евклидовом пространстве мы обозначаем угловыми скобками: $\langle u, v\rangle$, а их длины - прямыми: $|u|=\sqrt{\langle u, u\rangle}$. Направлением ненулевого вектора $u$ называется единичный вектор $u /|u|$.

ОПРЕДЕЛЕНИЕ ГРАДИЕНТА. Пусть $f$ - вогнутая функция на выпуклом подмножестве конечномерного евклидова пространства, а $x$ - внутренняя точка ее области определения. Градиентом функции $f$ в точке $x$ называется вектор $\operatorname{grad} f(x)$, в направлении которого производная функции $f$ в точке $x$ положительна и максимальна, а длина которого равна этой производной. Если же функция $f$ не возрастает ни в каком направлении, то ее градиент - нулевой вектор.

Вектор с такими свойствами существует, поскольку производная вогнутой функции в данной точке непрерывно зависит от направления. Кроме того, он единствен. В самом деле, пусть есть два различных направления, вдоль которых производные положительны и совпадают:

$$
f_{x}^{\prime}(u)=f_{x}^{\prime}(v)=b>0, \quad u \neq v, \quad|u|=|v|=1 .
$$

Тогда

$$
f_{x}^{\prime}(w) \geqslant b, \quad w=\frac{u}{2}+\frac{v}{2}
$$

поскольку производная вогнута. Поэтому $w \neq 0$, так как $f_{x}^{\prime}(0)=0$. Но

$$
f_{x}^{\prime}\left(\frac{w}{|w|}\right) \geqslant \frac{b}{|w|}>b
$$

поскольку $|w|<1$. То есть найдется направление, вдоль которого производная больше $b$. Иначе говоря, производная не может достигать своего положительного максимума в двух различных направлениях.

ЗАМЕчАНИЕ. В применении к гладкой функции данное выше определение дает ее обычный градиент. 
ЗАмЕчАниЕ. В отличие от гладких функций градиент суммы вогнутых функций не равен сумме их градиентов. Например, для функций на $\mathbb{R}$

$$
\operatorname{grad}(2 x)(0)=2, \quad \operatorname{grad}(-|x|)(0)=0, \quad \operatorname{grad}(2 x-|x|)(0)=1 .
$$

Основное свойство градиента. Следующее интуитивно очевидное свойство градиента вогнутой функции можно было бы взять за его определение.

ЛЕмма 1. Производная вогнутой функиии $f$ оценивается сверху линейной формой, сопряженной $\kappa$ ее градиенту, причем на самом градиенте неравенство превращается в равенство:

$$
f_{x}^{\prime}(v) \leqslant\langle\operatorname{grad} f(x), v\rangle \quad \forall v, \quad f_{x}^{\prime}(\operatorname{grad} f(x))=|\operatorname{grad} f(x)|^{2} .
$$

ДокАЗАтЕльство. Пусть $u_{0}=\operatorname{grad} f(x)$. Если $u_{0}=0$, то лемма очевидна. При $u_{0} \neq 0$ равенство из утверждения леммы верно, поскольку $\left|u_{0}\right|=$ $f_{x}^{\prime}\left(u_{0} /\left|u_{0}\right|\right)$ согласно определению градиента. Докажем неравенство при $u_{0} \neq 0$. Поскольку его левая часть вогнута, правая часть линейна, а само неравенство превращается в равенство при $v=u_{0}$, его достаточно проверить лишь в какойнибудь окрестности $u_{0}$, например в полупространстве, состоящем из всех таких $v$, что $\left\langle u_{0}, v\right\rangle>0$.

Для этого сначала рассмотрим гиперплоскость, перпендикулярную к градиенту и проходящую через его конец. А именно, пусть для некоторого $u_{1}$

$$
\left\langle u_{0}, u_{1}\right\rangle=\left|u_{0}\right|^{2}, \quad f_{x}^{\prime}\left(u_{1}\right)-\left\langle u_{0}, u_{1}\right\rangle=f_{x}^{\prime}\left(u_{1}\right)-\left|u_{0}\right|^{2}=b>0 .
$$

Тогда при $0 \leqslant \beta \leqslant 1$ из вогнутости производной получаем, что для вектора $u_{\beta}=(1-\beta) u_{0}+\beta u_{1}$, зависящего от $\beta$,

$$
f_{x}^{\prime}\left(u_{\beta}\right) \geqslant(1-\beta) f_{x}^{\prime}\left(u_{0}\right)+\beta f_{x}^{\prime}\left(u_{1}\right)=\left|u_{0}\right|^{2}+\beta b,
$$

поскольку $f_{x}^{\prime}\left(u_{0}\right)=\left|u_{0}\right|^{2}$ и $f_{x}^{\prime}\left(u_{1}\right)=\left|u_{0}\right|^{2}+b$. Поэтому

$$
\frac{f_{x}^{\prime}\left(u_{\beta}\right)}{\left|u_{\beta}\right|} \geqslant \frac{\left|u_{0}\right|^{2}+\beta b}{\left|u_{\beta}\right|}=\left|u_{0}\right|+\frac{\beta b}{\left|u_{0}\right|}+o(\beta), \quad \beta \rightarrow+0,
$$

поскольку $\left|u_{\beta}\right|=\left|u_{0}\right|+o(\beta)$. Иными словами, производная в направлении вектора $u_{\beta}$ при малых положительных $\beta$ превосходит длину градиента. Получаем противоречие с его определением.

Далее, поскольку обе части нашего неравенства однородны, оно верно не только для рассмотренной гиперплоскости, но и для открытого полупространства, состоящего из всех таких $v$, что $\left\langle u_{0}, v\right\rangle>0$.

В дальнейшем нам понадобится тот факт, что приращение вогнутой функции $f$ оценивается сверху линейной формой, сопряженной к ее градиенту:

$$
f(y)-f(x) \leqslant\langle\operatorname{grad} f(x), y-x\rangle .
$$

Это непосредственно следует из леммы 1 и неравенства (1). 


\section{3. Решения градиентных дифференциальных уравнений.}

Теорема 1. Пусть $f$ - вогнутая функиия на конечномерном евклидовом пространстве. Градиентное дифференциалъное уравнение

$$
\dot{x}=\operatorname{grad} f(x),
$$

где точка обозначает производную по времени справа, имеет непрерывное решение $x=\xi(t)$ :

$$
\lim _{\Delta t \rightarrow+0} \frac{\xi(t+\Delta t)-\xi(t)}{\Delta t}=\operatorname{grad} f(\xi(t)) \quad \forall t \geqslant 0
$$

при любом наперед заданном начальном условии

$$
\xi(0)=x_{0}
$$

Это решение единственно, поэтому определен фазовый поток $\mathbf{g}$ :

$$
\mathbf{g}_{f}^{t}\left(x_{0}\right)=\xi(t)
$$

который является непрерывной функиией всех своих аргументов: вогнутого потенциала $f$, начального условия $x_{0}$ и времени $t \geqslant 0$ в предположении, что пространство потенциалов снабжено компактно-открытой топологией.

\section{4. Доказательство существования, единственности и непрерыв- ной зависимости.}

Существование решений для гладких вогнутых потенциалов. Согласно общим теоремам о гладких дифференциальных уравнениях решение градиентного дифференциального уравнения с гладким потенциалом существует локально. Если потенциал еще и вогнутый, тогда любое решение неограниченно продолжается вперед по времени. Но не назад, как показывает пример $f(x)=-x^{4}$ градиентного дифференциального уравнения на прямой $x \in \mathbb{R}$.

Действительно, оценим производную по времени расстояния $|x|$ от решения нашего дифференциального уравнения до начала координат:

$$
|x|^{\cdot}=\frac{\langle\dot{x}, x\rangle}{|x|}=\frac{\langle\operatorname{grad} f(x), x\rangle}{|x|} \leqslant \frac{\langle\operatorname{grad} f(0), x\rangle}{|x|} \leqslant|\operatorname{grad} f(0)|,
$$

поскольку для любой вогнутой функции

$$
\langle\operatorname{grad} f(x), 0-x\rangle+\langle\operatorname{grad} f(0), x-0\rangle \geqslant(f(0)-f(x))+(f(x)-f(0))=0
$$

согласно неравенству (3). Поэтому решения нашего дифференциального уравнения не могут уходить в бесконечность при $t>0$ (но вполне могут при $t<0$ ). 
Существование решений для вогнутых однородных потенциалов. Потенциалы в следующей лемме уже необязательно гладкие.

ЛЕмма 2. Пусть функиия $f$ на конечномерном евклидовом пространстве вогнута и однородна степени 1:

$$
f(c x)=c f(x) \quad \forall c \geqslant 0 .
$$

Тогда кривая

$$
\xi(t)=t \operatorname{grad} f(0)
$$

является решением дифференциального уравнения

$$
\dot{x}=\operatorname{grad} f(x)
$$

с начальным условием $\xi(0)=0$ при всех $t \geqslant 0$.

ДокАзАТЕЛьСтво. Пусть $y=\operatorname{grad} f(0)$. Нам нужно доказать, что

$$
\operatorname{grad} f(t y)=y
$$

при всех $t>0$. Если $y=0$, то это очевидно. Предположим, что $y \neq 0$. Согласно основному свойству градиента (лемма 1)

$$
\begin{gathered}
f_{0}^{\prime}(t y)=t f_{0}^{\prime}(y)=|y|^{2} t \\
f_{0}^{\prime}(t y+v)-f_{0}^{\prime}(t y) \leqslant\langle y, t y+v\rangle-|y|^{2} t=\langle y, v\rangle
\end{gathered}
$$

при всех $v$. Но $f=f_{0}^{\prime}$, так как $f$ однородна. Поэтому

$$
f(t y)=|y|^{2} t, \quad f(t y+v)-f(t y) \leqslant\langle y, v\rangle \quad \forall v,
$$

откуда в соответствии с определением производной вытекает, что

$$
f_{t y}^{\prime}(y)=|y|^{2}, \quad f_{t y}^{\prime}(v) \leqslant\langle y, v\rangle \quad \forall v .
$$

Но это и означает, что максимум производной $f_{t y}^{\prime}$ достигается в направлении вектора $y$ и равен $|y|$.

Единственность и равномерно непрерывная зависимость. Следующая оценка доказывает единственность решения и его равномерно непрерывную зависимость от начального условия и потенциала, поскольку в качестве $M$ можно взять любое ограниченное открытое выпуклое множество, содержащее рассматриваемое решение.

Лемма 3. Рассмотрим на открытом выпуклом подмножестве $M$ конечномерного евклидова пространства два дифференциальных уравнения

$$
\dot{x}=\operatorname{grad} f(x), \quad \dot{y}=\operatorname{grad} g(y), \quad x, y \in M,
$$

с близкими вогнутыми потенциалами:

$$
|f(x)-g(x)| \leqslant \varepsilon \quad \forall x \in M .
$$


Тогда квадрат расстояния между их решениями $x=\xi(t)$ u $y=\eta(t)$ удовлетворяет неравенству

$$
R(t)=|\xi(t)-\eta(t)|^{2} \leqslant|\xi(0)-\eta(0)|^{2}+4 \varepsilon t
$$

при всех $t \geqslant 0$, пока они находятся внутри $M$.

ДокАЗАТЕЛьСтво. Оценим правую производную по времени квадрата расстояния между решениями, используя неравенство (3):

$$
\begin{aligned}
-\frac{1}{2} \dot{R} & =\langle\dot{x}-\dot{y}, y-x\rangle=\langle\operatorname{grad} f(x), y-x\rangle+\langle\operatorname{grad} g(y), x-y\rangle \\
& \geqslant(f(y)-f(x))+(g(x)-g(y)) .
\end{aligned}
$$

После перегруппировки получаем

$$
\dot{R} \leqslant 2(f(x)-g(x))+2(g(y)-f(y)) \leqslant 4 \varepsilon,
$$

так как потенциалы близки. Поэтому согласно лемме 4 функция $R(t)-4 \varepsilon t$ не возрастает и

$$
R(t)-4 \varepsilon t \leqslant R(0)
$$

при $t \geqslant 0$.

ЛЕмма 4. Функиия, непрерывная на промежутке и имеющая во всех его точках неположительную производную справа, не возрастает.

ДокАЗАТЕЛЬство. Пусть функция $r:[a, b] \rightarrow \mathbb{R}$, где $a<b$, непрерывна и $\dot{r}(c) \leqslant 0$ для любого $c \in[a, b)$. Тогда функция

$$
R(t)=r(t)-\frac{r(b)-r(a)}{b-a}(t-a)
$$

достигает своего минимума на отрезке $[a, b]$ в некоторой точке $c \in[a, b)$, поскольку $R(a)=R(b)$. Поэтому при $c<t<b$

$$
\frac{R(t)-R(c)}{t-c} \geqslant 0
$$

Следовательно,

$$
\dot{R}(c)=\lim _{t \rightarrow c+0} \frac{R(t)-R(c)}{t-c} \geqslant 0 .
$$

Поэтому

$$
\frac{r(b)-r(a)}{b-a}=\dot{r}(c)-\dot{R}(c) \leqslant 0,
$$

так как $\dot{r}(c) \leqslant 0$ и $\dot{R}(c) \geqslant 0$. Откуда вытекает, что $r(b) \leqslant r(a)$. 
Существование решений для всех вогнутых потенциалов. Попытаемся построить фазовый поток $\mathrm{g}$ из теоремы 1 следующим образом. Сначала определим его на всюду плотном подмножестве, содержащем все гладкие и все однородные вогнутые потенциалы, для всех начальных условий и всех $t \geqslant 0$. Согласно лемме 3 такой фазовый поток равномерно непрерывен, поэтому его можно единственным образом продолжить по непрерывности на все вогнутые потенциалы. Это единственное непрерывное продолжение и является искомым фазовым потоком $\mathbf{g}$, существование которого утверждается в теореме 1.

Опять-таки согласно лемме 3 , если решение $x=\xi(t)$ нашего дифференциального уравнения с начальным условием $x_{0}=\xi(0)$ существует, то оно дается только что построенным фазовым потоком

$$
\xi(t)=\mathbf{g}_{f}^{t}\left(x_{0}\right)
$$

при всех $t$ из промежутка, на котором оно определено. Однако априори фазовый поток g может давать "паразитную" траекторию, не являющуюся решением, если последнее не существует или определено не при всех $t \geqslant 0$. Следующая лемма показывает, что такого не происходит.

ЛЕмма 5. Пусть $f$-вогнутая функиия на конечномерном евклидовом пространстве. Тогда любая траектория $x=\mathbf{g}_{f}^{t}\left(x_{0}\right)$ толъко что построенного фазового потока при всех $t \geqslant 0$ является решением дифференииального уравнения $\dot{x}=\operatorname{grad} f(x)$.

ДокАЗАТЕЛьство. В двух словах идея доказательства такова. Посмотрим на окрестность какой-нибудь точки “в микроскоп". Тогда потенциал станет однородным и его градиент в рассматриваемой точке не изменится. Траектория же станет прямой, наклон которой равен производной исходной траектории в данной точке. Но градиент равен наклону прямой по лемме 2, значит, в рассматриваемой точке дифференциальное уравнение удовлетворяется.

Теперь более подробно. Наше градиентное дифференциальное уравнение инвариантно относительно следующих преобразований: прибавления постоянной к потенциалу, сдвига в пространстве, сдвига вперед по времени и одновременного растяжения графика потенциала, пространства и оси времени. Значит, и наш фазовый поток инвариантен относительно тех же преобразований. Действительно, на гладких потенциалах он является решением соответствующих дифференциальных уравнений и поэтому инвариантен, а на всех вогнутых потенциалах он тоже инвариантен по непрерывности. Описанная инвариантность выражается формулами:

1) прибавление постоянной к потенциалу: $\mathbf{g}_{f+\mathrm{const}}^{t}=\mathbf{g}_{f}^{t}$;

2) сдвиг пространства: $\mathbf{g}_{f\left(x-x_{0}\right)}^{t}=\mathbf{g}_{f(x)}^{t}+x_{0}$;

3) сдвиг вперед по времени: $\mathbf{g}_{f}^{t_{0}+t}=\mathbf{g}_{f}^{t} \circ \mathbf{g}_{f}^{t_{0}}, t_{0}, t \geqslant 0$;

4) одновременное растяжение графика потенциала, пространства и оси времени: $\mathbf{g}_{c f(x / c)}^{c t}\left(c x_{0}\right)=c \mathbf{g}_{f(x)}^{t}\left(x_{0}\right), c>0$.

Проверим, что наше дифференциальное уравнение удовлетворяется для траектории $\xi(t)=\mathbf{g}_{f}^{t}\left(x_{0}\right)$ при $t=t_{0}$. Согласно свойствам 2) и 3$)$ можно ограничиться случаем $t_{0}=0, x_{0}=0$. Используя свойство 1$)$, можно добиться, чтобы 
$f(0)=0$. Свойство 4) влечет, что

$$
c \xi\left(\frac{t}{c}\right)=\mathbf{g}_{c f(x / c)}^{t}(0) .
$$

Откуда, переходя к пределу с учетом непрерывности фазового потока, получаем

$$
\dot{\xi}(0)=\lim _{c \rightarrow+\infty} c \xi\left(\frac{1}{c}\right)=\mathbf{g}_{f_{0}^{\prime}}^{1}(0),
$$

поскольку $\xi(0)=x_{0}=0$ и при $c \rightarrow+\infty$

$$
c f\left(\frac{x}{c}\right) \rightarrow f_{0}^{\prime}(x)
$$

в компактно-открытой топологии, так как $f(0)=0$. (Последняя сходимость является следствием поточечной сходимости - см. п. 1.1.) Но

$$
\mathbf{g}_{f_{0}^{\prime}}^{1}(0)=\operatorname{grad} f_{0}^{\prime}(0)
$$

вследствие леммы 2. Наконец,

$$
\operatorname{grad} f_{0}^{\prime}(0)=\operatorname{grad} f(0)
$$

вследствие однородности производной $g=f_{0}^{\prime}$ и равенства $g_{0}^{\prime}=g$, справедливого для любой однородной функции $g$. Окончательно получаем

$$
\dot{\xi}(0)=\operatorname{grad} f(0),
$$

т.е. наше дифференциальное уравнение удовлетворяется при $t=0$.

1.5. Полный градиент относительно вогнутого потенциала, зависящего от времени. Как известно, градиент определен для всех гладких функций, а не только для вогнутых. Чтобы включить гладкие и вогнутые функции в единую теорию, мы рассматриваем их суммы, на которые определение и основное свойство градиента переносятся дословно, поскольку их производные вогнуты и однородны степени 1.

Кроме того, в приложениях потенциал может зависеть от времени, и в этом случае теорема существования решений градиентного дифференциального уравнения, вообще говоря, становится неверной. Оказывается, чтобы правильно сформулировать неавтономную версию теоремы 1, нужно определить так называемый полный градиент вогнутого потенциала. Если последний является гладкой функцией на пространстве-времени, то его градиент и полный градиент совпадают и полностью определяются поведением потенциала в данный момент времени. Полный же градиент негладкого вогнутого потенциала, вообще говоря, зависит не только от его поведения в данный момент времени, но и от его последующего поведения.

Относительная вогнУтость. Функция $\varphi$ на конечномерном вещественном линейном пространстве или его выпуклом подмножестве называется вогнутой относительно гладкой функиии е (или просто относительно вогнутой), если их разность $\varphi-e$ является вогнутой функцией. 
ОПРЕДЕЛЕНИЕ ПОЛНОГО ГРАДИЕНТА. Пусть $E$ - конечномерное евклидово пространство, $\varphi$ - относительно вогнутая функция, определенная на выпуклом подмножестве пространства-времени $E \times \mathbb{R}$, a $(x, t)$ - внутренняя мировая точка ее области определения. Полным градиентом функции $\varphi$ в мировой точке $(x, t)$ называется вектор $\operatorname{Grad} \varphi(x, t) \in E$, удовлетворяющий условию минимальности

$$
\frac{|v|^{2}}{2}-\varphi_{x, t}^{\prime}(v, 1) \rightarrow \min
$$

относительно $v \in E$.

Такой вектор существует и единствен, поскольку оптимизируемая величина непрерывна, ограничена снизу и строго выпукла по $v$. В пояснении нуждается только ограниченность снизу, которая следует из равенства

$$
\lim _{|v| \rightarrow+\infty} \frac{|v|^{2} / 2-\varphi_{x, t}^{\prime}(v, 1)}{|v|^{2}}=\frac{1}{2}-\lim _{|v| \rightarrow+\infty} \varphi_{x, t}^{\prime}\left(\frac{v}{|v|^{2}}, \frac{1}{|v|^{2}}\right)=\frac{1}{2}-\varphi_{x, t}^{\prime}(0)=\frac{1}{2},
$$

вытекающего из однородности производной по $v$.

ЗАмЕчАниЕ. В применении к гладкой функции сформулированное только что определение дает ее обычный градиент, для построения которого достаточно знать нашу функцию лишь в рассматриваемый момент времени. Точнее, полный градиент гладкой функции определяется ее производными вдоль пространственных векторов $(v, 0)$, поскольку в этом случае производная линейна:

$$
\varphi_{x, t}^{\prime}(v, 1)=\varphi_{x, t}^{\prime}(v, 0)+\varphi_{x, t}^{\prime}(0,1)
$$

а последнее слагаемое не зависит от $v$ и поэтому не влияет на условие минимальности. В случае же произвольной относительно вогнутой функции это уже не так - ее производная, вообе говоря, уже нелинейна, и полный градиент зависит от ее поведения как в данный момент времени, так и после него.

ЛЕмма 6. Если относительно вогнутая функция не зависит от времени, то ее полный градиент совпадает с градиентом, определенным в предыдущем napazpaøe.

ДокАЗАТЕЛЬСтво. Если $\varphi(x, t)=f(x)$, то условие минимальности полного градиента переписывается через длину и направление оптимизирующего вектора $v$ в следующем виде:

$$
\begin{aligned}
\min _{v}\left(\frac{|v|^{2}}{2}-\varphi_{x, t}^{\prime}(v, 1)\right) & =\min _{v}\left(\frac{|v|^{2}}{2}-f_{x}^{\prime}(v)\right)=\min _{|v| \geqslant 0} \min _{|\theta|=1}\left(\frac{|v|^{2}}{2}-|v| f_{x}^{\prime}(\theta)\right) \\
& =\min _{|v| \geqslant 0}\left(\frac{|v|^{2}}{2}-|v| \max _{|\theta|=1} f_{x}^{\prime}(\theta)\right) .
\end{aligned}
$$

Отсюда видно, что оно выполняется при

$$
|v|=\max \left\{0, \max _{|\theta|=1} f_{x}^{\prime}(\theta)\right\}, \quad f_{x}^{\prime}(\theta) \rightarrow \max ,
$$

т.е. при $v=\operatorname{grad} f(x)$ согласно определению градиента. 
Инвариантность полного градиента. Полный градиент в следующем естественном смысле инвариантен относительно сдвигов $x \mapsto x+\sigma(t)$ пространства, зависящих от времени. А именно, пусть

$$
\varphi^{\sigma}(x, t):=\langle\dot{\sigma}, x\rangle+\varphi(x-\sigma, t)
$$

тогда

$$
\operatorname{Grad} \varphi^{\sigma}(x+\sigma, t)=\dot{\sigma}+\operatorname{Grad} \varphi(x, t)
$$

В самом деле,

$\frac{|v|^{2}}{2}-\left(\varphi^{\sigma}\right)_{x+\sigma, t}^{\prime}(v, 1)=\frac{|v|^{2}}{2}-\langle\dot{\sigma}, v\rangle-\varphi_{x, t}^{\prime}(v-\dot{\sigma}, 1)=\frac{|v-\dot{\sigma}|^{2}}{2}-\frac{\dot{\sigma}^{2}}{2}-\varphi_{x, t}^{\prime}(v-\dot{\sigma}, 1)$,

откуда по условию минимальности из определения градиента мы и получаем требуемое равенство, поскольку член $-\dot{\sigma}^{2} / 2$ не зависит от $v$.

1.6. Основная теорема. Теорема 1 не применима в некоторых случаях, важных в приложениях. Например, часто бывает удобно рассматривать периодические потенциалы, но всякая периодическая вогнутая функция постоянна. А вот среди относительно вогнутых функций уже много периодических. Поэтому неавтономную версию теоремы 1 мы формулируем для относительно вогнутых потенциалов. При этом естественно становится возможным уход решений в бесконечность за конечное время, и поэтому теряется глобальное существование решений.

ТЕОРема 2. Пусть Е-конечномерное евклидово пространство, $\Pi \subset E \times \mathbb{R}$ - открытое выпуклое подмножество пространства-времени, а $\Phi_{\Pi, е}$ - семейство всех функииц на П, вогнутых относительно одной и той же гладкой

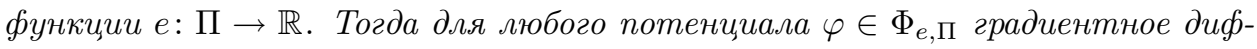
ференциальное уравнение

$$
\dot{x}=\operatorname{Grad} \varphi(x, t),
$$

где точка обозначает прочзводную по времени справа, при любом наперед заданном начальном условии

$$
\xi\left(t_{0}\right)=x_{0}, \quad\left(x_{0}, t_{0}\right) \in \Pi,
$$

имеет непрерывное решение $x=\xi(t)$ на некотором полуинтервале:

$$
\lim _{\Delta t \rightarrow+0} \frac{\xi(t+\Delta t)-\xi(t)}{\Delta t}=\operatorname{Grad} \varphi(\xi(t), t) \quad \forall t \in\left[t_{0}, t_{0}+\delta\right), \quad \delta>0 .
$$

Такое решение единственно в том смысле, что любые два решения с одним и тем же начальным условием, но определенные на, быть может, разных полуинтервалах, совпадают на их пересечении. Кроме того, значение решения $\xi(t)$ непрерывно зависит от времени $t \geqslant t_{0}$, начального условия $\left(t_{0}, x_{0}\right)$ и потен-

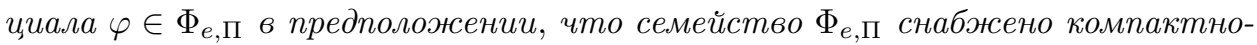
открытой топологией. 
1.7. Доказательство основной теоремы. Доказательство теоремы 2 проводится по той же схеме, что и доказательство теоремы 1 - только уже не надо заботиться о бесконечном продолжении решений вперед по времени.

Существование решений для гладких потенциалов. Если потенциал $\varphi$ - гладкая функция, то решение заведомо существует локально согласно общей теории обыкновенных дифференциальных уравнений.

Существование решений для вогнутых однородных потенциалов. Если функция $\varphi: E \times \mathbb{R} \rightarrow \mathbb{R}$ вогнута и однородна степени 1 :

$$
\varphi(c x, c t)=c \varphi(x, t) \quad \forall c \geqslant 0,
$$

то кривая

$$
\xi(t)=t \operatorname{Grad} \varphi(0,0)
$$

является решением дифференциального уравнения

$$
\dot{x}=\operatorname{Grad} \varphi(x, t)
$$

с начальным условием $\xi(0)=0$ при всех $t \geqslant 0$. Это прямое следствие теоремы 3 .

Единственность и равномерно непрерывная зависимость. Как и в автономном случае, рассмотрим на открытом выпуклом подмножестве $M \subset E \times \mathbb{R}$ пространства-времени два дифференциальных уравнения

$$
\dot{x}=\operatorname{Grad} \varphi(x, t), \quad \dot{y}=\operatorname{Grad} \psi(y, t)
$$

с близкими потенциалами

$$
|\varphi(x, t)-\psi(x, t)| \leqslant \varepsilon \quad \forall(x, t) \in M,
$$

вогнутыми относительно гладкой функции $A|x|^{2}+A t^{2}$. Тогда квадрат расстояния между решениями $x=\xi(t)$ и $y=\eta(t)$ наших дифференциальных уравнений удовлетворяет неравенству

$$
R(t)=|\xi(t)-\eta(t)|^{2} \leqslant \varrho\left(R_{0}, \varepsilon, t\right), \quad \text { где } \quad R_{0}=\left|\xi\left(t_{0}\right)-\eta\left(t_{0}\right)\right|^{2},
$$

при всех $t \geqslant t_{0}$, пока они находятся внутри $M$; функция

$$
\varrho\left(R_{0}, \varepsilon, t\right)=R_{0} e^{4 A\left(t-t_{0}\right)}+\varepsilon \frac{e^{4 A\left(t-t_{0}\right)}-1}{A}
$$

- единственное решение линейного дифференциального уравнения $\dot{\varrho}=4 \varepsilon+4 A \varrho$ с начальным условием $\varrho\left(R_{0}, \varepsilon, t_{0}\right)=R_{0}$. В частности, при $A=0$

$$
\varrho\left(R_{0}, \varepsilon, t\right)=R_{0}+4 \varepsilon\left(t-t_{0}\right) .
$$

Эта оценка доказывает единственность решения и его равномерно непрерывную зависимость от начального условия и потенциала. Действительно, в качестве $M$ можно взять любое ограниченное открытое выпуклое множество, содержащее рассматриваемое решение, замыкание $\bar{M}$ которого содержится в П, 
а в качестве $A$ - максимум на $\bar{M}$ второй производной гладкой функции $e$ по всем направлениям в пространстве-времени.

Для доказательства нашей оценки сначала используем неравенство (1):

$$
\begin{aligned}
\varphi(y, t)-\varphi(x, t) & =f(y, t)+A|y|^{2}-f(x, t)-A|x|^{2} \leqslant f_{x, t}^{\prime}(y-x, 0)+A|y|^{2}-A|x|^{2} \\
& =\varphi_{x, t}^{\prime}(y-x, 0)+A|y-x|^{2}
\end{aligned}
$$

откуда согласно доказанной ниже оценке (9) получаем

$$
\varphi(y, t)-\varphi(x, t) \leqslant\langle\operatorname{Grad} \varphi(x, t), y-x\rangle+A|y-x|^{2} .
$$

Как и в автономном случае, оценим теперь правую производную по времени квадрата расстояния между решениями:

$$
\begin{aligned}
-\frac{1}{2} \dot{R} & =\langle\dot{x}-\dot{y}, y-x\rangle=\langle\operatorname{Grad} \varphi(x, t), y-x\rangle+\langle\operatorname{Grad} \psi(y, t), x-y\rangle \\
& \geqslant\left(\varphi(y, t)-\varphi(x, t)-A|y-x|^{2}\right)+\left(\psi(x, t)-\psi(y, t)-A|x-y|^{2}\right) .
\end{aligned}
$$

После перегруппировки получаем

$$
\dot{R} \leqslant 2(\varphi(x, t)-\psi(x, t))+2(\psi(y, t)-\varphi(y, t))+4 A|x-y|^{2} \leqslant 4 \varepsilon+4 A R,
$$

так как наши потенциалы близки. Поэтому согласно лемме 4 функция

$$
r(t)=e^{-4 A t}\left(R(t)-\varrho\left(R_{0}, \varepsilon, t\right)\right)
$$

не возрастает, поскольку

$$
e^{4 A t} \dot{r}=-4 A R+4 A \varrho+\dot{R}-\dot{\varrho} \leqslant 0
$$

И

$$
r(t)=e^{-4 A t}\left(R(t)-\varrho\left(R_{0}, \varepsilon, t\right)\right) \leqslant r(0)=0
$$

при $t \geqslant 0$, что и дает требуемую оценку $R(t) \leqslant \varrho\left(R_{0}, \varepsilon, t\right)$.

Существование решений для относительно вогнутых потенциалов. Рассуждения те же, что и в автономном случае. А именно, отображение

$$
\mathrm{g}:\left(\varphi, x_{0}, t\right) \mapsto \xi(t)
$$

определено на гладких и однородных вогнутых потенциалах. Продолжим его по непрерывности на относительно вогнутые потенциалы и проверим, что его траектории являются решениями дифференциального уравнения. Действительно, посмотрим на окрестность какой-нибудь мировой точки в "микроскоп". Проходящая через нее траектория выпрямляется, а ее наклон совпадает с производной исходной траектории в рассматриваемой точке. Потенциал же становится однородным, а его полный градиент остается прежним. Но для однородного потенциала наклон выпрямленной траектории совпадает с его градиентом, значит, исходная траектория удовлетворяет дифференциальному уравнению в рассматриваемой точке. 


\section{§ 2. Траектории принципа наименьшего действия}

2.1. Вариационная задача. В приложениях относительно вогнутые функции возникают как решения вариационных задач с гладкими данными. Рассмотрим, например, задачу о наименьшем действии $\varphi$ в $n$-мерном пространстве:

$$
\begin{gathered}
\varphi(x, t)=\min _{\gamma(t)=x} W_{t}[\gamma], \quad W_{t}[\gamma]=\varphi_{0}(\gamma(0))+\int_{0}^{t} L(\dot{\gamma}(\tau), \gamma(\tau), \tau) d \tau, \\
L(\dot{x}, x, t)=\frac{|\dot{x}|^{2}}{2}-U(x, t), \quad x=\left(x_{1}, \ldots, x_{n}\right) \in \mathbb{R}^{n}, \quad t \in \mathbb{R}, \quad \gamma:[0, t] \rightarrow \mathbb{R}^{n},
\end{gathered}
$$

лагранжиан которой имеет естественный механический смысл - это просто разность кинетической энергии частицы и ее потенциальной энергии во внешнем силовом поле

$$
F(x, t)=-\partial_{x} U(x, t), \quad \partial_{x}=\left(\partial_{x_{1}}, \ldots, \partial_{x_{n}}\right),
$$

начальное действие $\varphi_{0}$ и потенциальная энергия $U$ - гладкие периодические по $x$ функции, а минимум берется среди всех непрерывных траекторий $\gamma$, имеющих кусочно непрерывные производные и заканчивающихся в точке $x$.

Траектории $\gamma_{\min }:[0, t] \rightarrow \mathbb{R}^{n}$, вдоль которых достигается минимум действия, называются оптимальными. Хорошо известно, что они являются траекториями частиц, двигающихся во внешнем силовом поле с начальной скоростью, равной градиенту начального действия:

$$
\ddot{\gamma}_{\min }(t)=F\left(\gamma_{\min }(t), t\right), \quad \dot{\gamma}_{\min }(0)=\partial_{x} \varphi_{0}\left(\gamma_{\min }(0)\right) .
$$

Более того, в тех мировых точках, где заканчивается только одна оптимальная траектория, наименьшее действие $\varphi$ имеет производную и удовлетворяет уравнению Гамильтона-Якоби, а его градиент равен скорости частицы в рассматриваемой мировой точке:

$$
\partial_{t} \varphi+H\left(\partial_{x} \varphi, x, t\right)=0, \quad H(p, x, t)=\frac{|p|^{2}}{2}+U(x, t), \quad \dot{\gamma}_{\min }(t)=\partial_{x} \varphi\left(\gamma_{\min }(t), t\right) .
$$

Однако в некоторых мировых точках могут заканчиваться две или более разных оптимальных траекторий, значения действия вдоль которых само собой совпадают. Такие точки образуют ударную волну

$$
S_{t}=\left\{x \in \mathbb{R}^{n} \mid x=\gamma_{\min }^{1}(t)=\gamma_{\min }^{2}(t), \gamma_{\min }^{1}(0) \neq \gamma_{\min }^{2}(0)\right\},
$$

а поле скоростей $\partial_{x} \varphi$ в них разрывно, поскольку разные траектории $\gamma_{\min }^{1}$ и $\gamma_{\min }^{2}$ внешнего силового поля $F$ приходят в одну и ту же точку $(x, t)$ с разными скоростями $\dot{\gamma}_{\min }^{1}(t) \neq \dot{\gamma}_{\min }^{2}(t)$. С течением времени $t$ ударная волна $S_{t}$ испытывает перестройки (см. рис. 4).

Классический пример появления разрыва у поля скоростей при $n=1$ и $U \equiv 0$ - это следующее решение нашей вариационной задачи

$$
\varphi(x, t)=\min _{\beta}\left\{\frac{\beta^{4}}{4}+(1-t) \frac{\beta^{2}}{2}+x \beta\right\},
$$

которое не является гладким при $x=0$ и $t \geqslant 1$. 
Итак, даже при гладких периодических данных наименьшее действие $\varphi$ хоть и непрерывно, но поле скоростей частиц, равное его обычному градиенту, разрывно на ударной волне, в точках которой заканчиваются по меньшей мере две оптимальные траектории, т.е. происходят столкновения частиц.

Исчезающая вязкость. В физике наименьшее действие возникает как предел (при исчезновении вязкости) потенциала скоростей бесконечно сжимаемой вязкой жидкости, текущей во внешнем потенциальном силовом поле. Даже при отсутствии внешнего силового поля теория такой жидкости оказывается весьма содержательной и важной для приложений (см., например, [3], [4]).

Зависящий от вязкости $\nu$ потенциал $\psi^{\nu}(x, t)$ поля скоростей бесконечно сжимаемой вязкой жидкости является решением задачи Коши

$$
\partial_{t} \psi^{\nu}+H\left(\partial_{x} \psi^{\nu}, x, t\right)=\nu \Delta_{x} \psi^{\nu}, \quad \nu>0, \quad \psi^{\nu}(x, 0)=\varphi_{0}(x),
$$

где $\Delta_{x}=\left\langle\partial_{x}, \partial_{x}\right\rangle$ - лапласиан в $x$-пространстве. Оказывается, что при исчезновении вязкости потенциал поля скоростей стремится к наименьшему действию в нашей вариационной задаче:

$$
\varphi(x, t)=\lim _{\nu \rightarrow+0} \psi^{\nu}(x, t)
$$

Доказывается этот факт так. При отсутствии внешней силы задача (4) допускает явное решение, которое и дает указанную асимптотику (см., например, [3]). При наличии же внешней силы доказательство сложнее и опирается на теорию обобщенных решений многомерного уравнения Гамильтона-Якоби, построенную в [5]-[7]. Его можно, например, извлечь из более общего результата [8], а вкратце идея такова. Докажем сначала, что и асимптотика, и наименьшее действие являются обобщенными решениями уравнения ГамильтонаЯкоби в смысле [5] или [7], а затем применим теорему единственности для таких обобщенных решений.

Относительная вогнутость наименьшего действия. Как было отмечено выше, наименьшее действие в нашей вариационной задаче не обязано быть гладким. Зато оно относительно вогнуто, поскольку на конечном отрезке времени его можно представить как минимум компактного семейства гладких функций

$$
\varphi(x, t)=\min _{\beta \in K_{T}} P_{\beta}(x, t) \quad \forall(x, t) \in \mathbb{R}^{n} \times[0, T],
$$

где $0<T<+\infty$, а $P_{\beta}$ - гладкая функция от $x$ и $t$, периодическая по $x$ и гладко зависящая от параметра $\beta$, по компактному множеству $K_{T}$ значений которого и берется минимум.

Для построения подходящего семейства $P$ вспомним, что минимум действия обязательно достигается на каком-то решении уравнения Ньютона, которое однозначно определяется своими начальными условиями в момент времени $t$ :

$$
\ddot{\gamma}_{\beta}(\tau)=F\left(\gamma_{\beta}(\tau), \tau\right), \quad \gamma_{\beta}(t)=x, \quad \dot{\gamma}_{\beta}(t)=\beta .
$$


Пусть теперь значение оптимизируемого функционала на этом решении $\gamma_{\beta}$, зависящем от скорости $\beta$, и есть $P_{\beta}(x, t)$, которое можно представить в виде

$$
P_{\beta}(x, t)=\varphi_{0}(x)+t Q_{\beta}(x, t),
$$

где $Q$ - гладкая функция от $x, t$ и $\beta$, периодическая по $x$, поскольку $P_{\beta}(x, 0)=$ $\varphi_{0}(x)$. Но при взятии минимума достаточно ограничиться значениями $\beta$ из какого-то компактного множества $K_{T}$, поскольку при больших $|\beta|$ главный вклад в действие вносит кинетическая энергия частицы

$$
Q_{\beta}(x, t)=\frac{|\beta|^{2}}{2}+O(|\beta|), \quad|\beta| \rightarrow+\infty,
$$

причем эта асимптотика равномерна по $x$ и $t \in[0, T]$.

Докажем эту асимптотику. Для решения уравнения Ньютона $\gamma_{\beta}$ справедливы равномерные по $x, t \in[0, T]$ и $\tau \in[0, t]$ асимптотики

$$
\gamma_{\beta}(\tau)=x+(\tau-t) \beta+t O(1), \quad \dot{\gamma}_{\beta}(\tau)=\beta+O(1), \quad|\beta| \rightarrow+\infty .
$$

Они доказываются введением новой траектории $\sigma(\varsigma)$,

$$
\varsigma=\frac{\tau}{t} \in[0,1], \quad \sigma_{\beta}(\varsigma)=\frac{\gamma_{\beta}(t \varsigma)-x}{|\beta| t}, \quad \dot{\sigma}_{\beta}(\varsigma)=\frac{\dot{\gamma}_{\beta}(t \varsigma)}{|\beta|},
$$

удовлетворяющей уравнению

$$
\ddot{\sigma}_{\beta}(\varsigma)=\frac{t \ddot{\gamma}_{\beta}(t \varsigma)}{|\beta|}=\frac{t F\left(x+|\beta| t \sigma_{\beta}(\varsigma), t \varsigma\right)}{|\beta|}=O\left(\frac{1}{|\beta|}\right), \quad|\beta| \rightarrow+\infty,
$$

и начальным условиям $\sigma_{\beta}(1)=0, \dot{\sigma}_{\beta}(1)=\beta /|\beta|$. Поэтому

$$
\sigma_{\beta}(\varsigma)=(\varsigma-1) \frac{\beta}{|\beta|}+O\left(\frac{1}{|\beta|}\right), \quad \dot{\sigma}_{\beta}(\varsigma)=\frac{\beta}{|\beta|}+O\left(\frac{1}{|\beta|}\right), \quad|\beta| \rightarrow+\infty .
$$

Возвращаясь теперь к старой траектории $\gamma_{\beta}(\tau)$, получаем для нее требуемые асимптотики, подставляя которые в оптимизируемый функционал

$$
\begin{aligned}
Q_{\beta}(x, t)= & \frac{\varphi_{0}\left(\gamma_{\beta}(0)\right)-\varphi_{0}(x)}{t}+\frac{1}{t} \int_{0}^{t} L\left(\dot{\gamma}_{\beta}(\tau), \gamma_{\beta}(\tau), \tau\right) d \tau \\
= & \frac{\varphi_{0}(x-t \beta+t O(1))-\varphi_{0}(x)}{t}+\frac{1}{t} \int_{0}^{t} \frac{|\beta+O(1)|^{2}}{2} d \tau \\
& -\frac{1}{t} \int_{0}^{t} U(x+(\tau-t) \beta+t O(1), \tau) d \tau
\end{aligned}
$$

получаем указанную асимптотику для $Q_{\beta}$.

Движение частиц. Поскольку наименьшее действие относительно вогнуто, вышеизложенные результаты позволяют корректно определить движение частиц и внутри ударной волны, если в качестве скорости частицы взять полный 
градиент наименьшего действия. А траекториями частиц естественно объявить решения дифференциального уравнения

$$
\dot{\xi}(t)=\operatorname{Grad} \varphi(\xi(t), t),
$$

удовлетворяющие теоремам существования и единственности в силу теоремы 2 . Согласно этой картине на ударной волне происходят столкновения частиц, после чего они слипаются и в дальнейшем двигаются как одно целое, т.е. образуется массивная частица. Последняя как-то перемещается по ударной волне, а с ней постоянно сталкиваются и слипаются все новые и новые частицы.

Массивные частицы никогда не покидают ударную волну, поскольку вне нее движение происходит по оптимальным траекториям, которые могут лишь заканчиваться в ударной волне, но не проходить через нее. Действительно, если вдоль траектории $\gamma_{\min }:[0, t] \rightarrow \mathbb{R}^{n}$ достигается единственный минимум действия среди всех траекторий, заканчивающихся в мировой точке $\left(\gamma_{\min }(t), t\right)$, то при любом $\tau<t$ минимум действия среди всех траекторий, заканчивающихся в мировой точке $\left(\gamma_{\min }(\tau), \tau\right)$, достигается вдоль начальной части

$$
\left.\gamma_{\min }\right|_{[0, \tau]}:[0, \tau] \rightarrow \mathbb{R}^{n}
$$

исходной оптимальной траектории $\gamma_{\min }$ и этот минимум единствен. (Иначе действие вдоль $\gamma_{\min }$ не являлось бы единственным минимумом, поскольку оно бы уменьшалось или оставалось прежним после некоторого изменения траектории на отрезке $[0, \tau]$.)

Физическая естественность. Оказывается, что построенное нами движение частиц физически оправдано введением исчезающей вязкости. А именно, пусть $\eta^{\nu}$ - решение задачи Коши (4), зависящее от вязкости $\nu$ и периодическое по $x$. Хорошо известно, что при положительной вязкости оно является еще и гладким, поэтому дифференциальное уравнение

$$
\dot{\eta}^{\nu}(t)=\operatorname{Grad} \psi^{\nu}\left(\eta^{\nu}(t), t\right)
$$

корректно определяет траектории частиц бесконечно сжимаемой вязкой жидкости. Оказывается, что при стремлении вязкости к нулю эти траектории сходятся к построенным выше траекториям нашей вариационной задачи, т.е. к решениям дифференциального уравнения (7):

$$
\eta^{\nu}(t) \rightarrow \xi(t) \quad \text { при } \quad \nu \rightarrow+0 \quad \text { и } \quad \eta^{\nu}(0) \rightarrow \xi(0) .
$$

В самом деле, как будет показано ниже, для любого $T>0$ найдется такая постоянная $C$, что при всех $\nu>0$ в полосе $\Pi=\mathbb{R}^{n} \times[0, T]$ потенциалы $\psi^{\nu}$ вогнуты относительно гладкой функции $e(x, t)=C\left(|x|^{2}+t^{2}\right)$. Иначе говоря, все $\psi^{\nu} \in \Phi_{e, \Pi}$. Но поточечная сходимость (5) в пространстве $\Phi_{e, \Pi}$ обязательно рав-

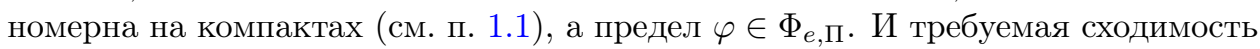
траекторий следует из основной теоремы 2. 
Существование же постоянной $C$ - это просто равномерная ограниченность сверху второй производной потенциала $\psi^{\nu}$ в нашей полосе П, которая следует из принципа максимума. А именно, обозначим через

$$
\partial_{\theta}=\left\langle v, \partial_{x}\right\rangle+s \partial_{t}=\sum_{i=1}^{n} v_{i} \partial_{x_{i}}+s \partial_{t}, \quad \text { где } \quad \theta=(v, s) \in S^{n}=\left\{|v|^{2}+s^{2}=1\right\}
$$

оператор дифференцирования вдоль направления в пространстве-времени. Применяя его дважды к (4), получаем

$$
\partial_{t}\left(\partial_{\theta}^{2} \psi^{\nu}\right)+\left|\partial_{x}\left(\partial_{\theta} \psi^{\nu}\right)\right|^{2}+\left\langle\partial_{x} \psi^{\nu}, \partial_{x}\left(\partial_{\theta}^{2} \psi^{\nu}\right)\right\rangle+\partial_{\theta}^{2} U=\nu \Delta_{x}\left(\partial_{\theta}^{2} \psi^{\nu}\right)
$$

что влечет неравенство

$$
\partial_{t}\left(\partial_{\theta}^{2} \psi^{\nu}\right)+\left\langle\partial_{x} \psi^{\nu}, \partial_{x}\left(\partial_{\theta}^{2} \psi^{\nu}\right)\right\rangle+C_{1} \leqslant \nu \Delta_{x}\left(\partial_{\theta}^{2} \psi^{\nu}\right)
$$

где

$$
C_{1}=\min _{\theta \in S^{n}} \min _{(x, t) \in \Pi} \partial_{\theta}^{2} U(x, t)
$$

- постоянная, зависящая от $T$ и $U$. Поэтому справедливо неравенство

$$
\partial_{t} \omega+\left\langle\partial_{x} \psi^{\nu}, \partial_{x} \omega\right\rangle \leqslant \nu \Delta_{x} \omega, \quad \text { где } \omega(\theta, x, t)=\partial_{\theta}^{2} \psi^{\nu}(x, t)+C_{1} t .
$$

Таким образом, в точке максимума $\omega$ выполняется неравенство $\partial_{t} \omega \leqslant 0$, означающее, что максимум достигается при $t=0$ :

$$
\omega(\theta, x, t) \leqslant \max _{x \in \mathbb{R}^{n}} \omega(\theta, x, 0) \leqslant C_{2},
$$

где

$$
C_{2}=\max _{\theta \in S^{n}} \max _{x \in \mathbb{R}^{n}} \omega(\theta, x, 0)=\left.\max _{\theta \in S^{n}} \max _{x \in \mathbb{R}^{n}} \partial_{\theta}^{2} \psi^{\nu}\right|_{t=0}
$$

- зависящая от $\varphi_{0}$ и $U$ постоянная, которая существует, поскольку согласно уравнению (4) временны́е производные потенциала $\psi^{\nu}$ выражаются через его пространственные производные, потенциальную энергию $U$ и ее производные. Окончательно получаем:

$$
\partial_{\theta}^{2} \psi^{\nu}(x, t)=\omega(\theta, x, t)-C_{1} t \leqslant C_{2}-C_{1} t \leqslant C, \quad \text { где } \quad C=\max \left\{C_{2}, C_{2}-C_{1} T\right\} .
$$

Скорости частиц. Каким образом можно найти поле предельных скоростей $u(x, t)=\operatorname{Grad} \varphi(x, t) ?$ Оказывается, что оно удовлетворяет следующему правилу, позволяющему эффективно его вычислять. А именно, скорость частицы это центр шара минимально возможного радиуса, который содержит скорости всех оптимальных траекторий, заканчивающихся в точке, где находится частица в рассматриваемый момент времени. Иначе говоря, скорость частицы это центр минимального шара, содержащего скорости всех частиц, бесконечно близких к данной в рассматриваемый момент времени.

В самом деле, пусть $\mathscr{V}_{x, t}$ - это множество скоростей $\dot{\gamma}_{\min }(t)$ всех оптимальных траекторий, заканчивающихся в мировой точке $(x, t)$, т.е. таких, что $\gamma_{\min }(t)=x$. Тогда

$$
\varphi_{x, t}^{\prime}(w, 0)=\min _{v \in \mathscr{V}_{x, t}}\langle v, w\rangle
$$


согласно хорошо известному соотношению

$$
W_{t}\left[\gamma_{\min }+c \Delta \gamma\right]=W_{t}\left[\gamma_{\min }\right]+c\left\langle\dot{\gamma}_{\min }(t), \Delta \gamma(t)\right\rangle+o(c), \quad c \rightarrow 0,
$$

справедливому для любой гладкой траектории $\Delta \gamma:[0, t] \rightarrow \mathbb{R}^{n}$. Но при $s \geqslant 0$ для производной наименьшего действия справедлива формула

$$
\varphi_{x, t}^{\prime}(w, s)=\min _{\beta}\left\{\varphi_{x, t}^{\prime}(w-\beta s, 0)+s\left(\frac{|\beta|^{2}}{2}-U(x, t)\right)\right\},
$$

поскольку эта производная сама является решением замороженной в точке $(x, t)$ нашей вариационной задачи. Объединяя две только что приведенные формулы для производной наименьшего действия, получаем

$$
\begin{aligned}
\varphi_{x, t}^{\prime}(w, 1) & =\min _{\beta} \min _{v \in \mathscr{V}_{x, t}}\left\{\langle v, w-\beta\rangle+\frac{|\beta|^{2}}{2}\right\}-U(x, t) \\
& =\min _{v \in \mathscr{V}_{x, t}}\left\{\langle v, w-v\rangle+\frac{|v|^{2}}{2}\right\}-U(x, t)=\min _{v \in \mathscr{V}_{x, t}}\left\{\langle v, w\rangle-\frac{|v|^{2}}{2}\right\}-U(x, t) .
\end{aligned}
$$

Но полный градиент $\operatorname{Grad} \varphi(x, t)$ по своему определению минимизирует величину

$$
\begin{aligned}
\frac{|w|^{2}}{2}-\varphi_{x, t}^{\prime}(w, 1) & =\max _{v \in \mathscr{V}_{x, t}}\left\{\frac{|w|^{2}}{2}-\langle v, w\rangle+\frac{|v|^{2}}{2}\right\}+U(x, t) \\
& =\frac{1}{2} \max _{v \in \mathscr{V}_{x, t}}\left\{|v-w|^{2}\right\}+U(x, t),
\end{aligned}
$$

которая достигает своего минимума как раз в центре минимального шара, содержащего множество скоростей $\mathscr{V}_{x, t}$. Значит, этот центр и есть искомый полный градиент.

Кластеры на плоскости. Объясним теперь, как получаются типичные случаи взаимодействия кластеров с узлами ударной волны, изображенные на рис. 5-9.

Рисунок 5 получается так. Производная наименьшего действия в узле $(x, t)$ является минимумом трех линейных решений замороженной вариационной задачи

$$
\varphi_{x, t}^{\prime}(w, s)=\min _{i=1,2,3}\left\{\left\langle v_{i}, w\right\rangle-s\left(\frac{\left|v_{i}\right|^{2}}{2}+U(x, t)\right)\right\}
$$

со скоростями $v_{1}, v_{2}$ и $v_{3}$, не лежащими на одной прямой. Скорость узла $V$ равна центру окружности, проходящей через скорости $v_{1}, v_{2}$ и $v_{3}$, поскольку эта скорость определяется из условия равенства всех трех линейных решений на мировом векторе $(V, 1)$ :

$$
-\frac{\left|v_{1}-V\right|^{2}}{2}=-\frac{\left|v_{2}-V\right|^{2}}{2}=-\frac{\left|v_{3}-V\right|^{2}}{2},
$$

которое здесь выписано с точностью до общего слагаемого $V^{2} / 2-U(x, t)$. Направления же $N$ ударных волн, выходящих из узла, находятся из условия, когда два линейных решения совпадают и меньше третьего на мировых векторах $(N, 0)$ :

$$
\left\langle v_{i}, N\right\rangle=\left\langle v_{j}, N\right\rangle\left\langle\left\langle v_{k}, N\right\rangle, \quad\{i, j, k\}=\{1,2,3\}\right.
$$


А согласно вышеизложенному скорость $u(x, t)$ частицы в узле равна центру круга минимального радиуса, содержащего скорости $v_{1}, v_{2}$ и $v_{3}$.

Перейдем теперь в систему отсчета, связанную с узлом, т.е. в которой $V=0$. Если треугольник $\triangle v_{1} v_{2} v_{3}$ остроугольный, то $u(x, t)=0$ и получается притягивающий узел, изображенный на рис. 5 справа. В этом случае $\varphi(\cdot, t)$ достигает в узле локального максимума. Если же треугольник $\triangle v_{1} v_{2} v_{3}$ тупоугольный, то $u(x, t) \neq 0$ и получается нейтральный узел, изображенный на рис. 5 слева, а узел уже не является точкой максимума мгновенного наименьшего действия $\varphi(\cdot, t)$.

Вторые и третьи перестройки на рис. 6-9 получаются следующим образом. Производная наименьшего действия в мировой точке $(x, t)$ 5-й или 6 -й перестройки рис. 4 является минимумом четырех линейных решений замороженной вариационной задачи

$$
\varphi_{x, t}^{\prime}(w, s)=\min _{i=1,2,3,4}\left\{\left\langle v_{i}, w\right\rangle-s\left(\frac{\left|v_{i}\right|^{2}}{2}+U(x, t)\right)\right\}
$$

со скоростями $v_{1}, v_{2}, v_{3}$ и $v_{4}$, не лежащими на одной окружности, никакие три из которых не лежат на одной прямой.

Конус в пространстве $(w, s)$, состоящий из касательных к ударной волне лучей с началом в мировой точке перестройки, находится из условия, когда два линейных решения совпадают и не больше каждого из двух других. Сечения этого конуса при $s=$ const $<0, s=0$ и $s=$ const $>0$ и дают рисунки локальной ударной волны соответственно до, в момент и после перестройки.

Эти сечения находятся так. Сечение $s=\mathrm{const} \geqslant 0$ получается умножением на $s$ из множества точек, расстояние от каждой из которых до скоростей $v_{1}$, $v_{2}, v_{3}$ и $v_{4}$ достигает своего максимума по меньшей мере для двух скоростей. Сечение $s=$ const $\leqslant 0$ получается умножением на $s$ из множества точек, расстояние от каждой из которых до скоростей $v_{1}, v_{2}, v_{3}$ и $v_{4}$ достигает своего минимума по меньшей мере для двух скоростей.

В частности, если выпуклая оболочка этих четырех скоростей - треугольник, то получается 5-я перестройка на рис. 4, если четырехугольник - то 6-я.

Назовем набор из четырех скоростей $v_{1}, v_{2}, v_{3}$ и $v_{4}$ на плоскости типичным, если никакие три из них не лежат на одной прямой, все четыре не лежат на одной окружности, а ни один из четырех треугольников с вершинами в них не является прямоугольным.

В случае общего положения производная наименьшего действия в точке перестройки определяется типичным набором скоростей. Каждый из четырех треугольников, образованных типичным набором из четырех скоростей, соответствует узлу. Если треугольник остроугольный, то получается притягивающий узел, если тупоугольный - то нейтральный. Если четвертая скорость находится вне окружности, проходящей через вершины треугольника, то узел входит в перестройку, если внутри - то выходит. Скорость частиц, вылетевших из точки перестройки, равна центру минимального круга, содержащего все четыре скорости. 
Пространство типичных неупорядоченных наборов скоростей состоит из нескольких компонент связности, среди которых имеется 15 компонент, не переходящих друг в друга при осевой симметрии. Представители этих компонент изображены на рис. 10 и 11, на которых тупые углы отмечены дугами со стрелками, за исключением случаев, когда один тупой угол содержит другой, в этих случаях бо́льшие углы мы не отмечаем. Все остальные неотмеченные углы являются острыми.
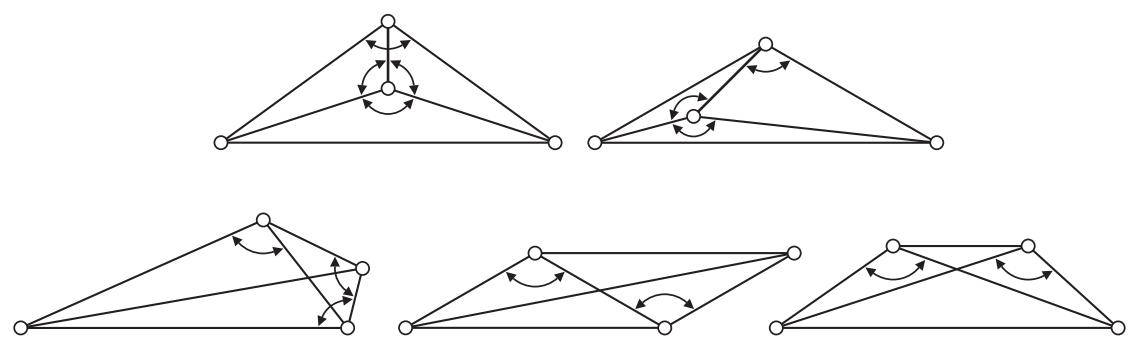

Рис. 10. Типичные наборы скоростей, определяющие перестройки лишь с нейтральными узлами

На рис. 10 изображены представители пяти компонент связности, в которых все четыре треугольника являются тупоугольными. Эти наборы определяют перестройки, в которых все узлы нейтральные. Поэтому кластеры в них не участвуют, и мы этими компонентами не интересуемся. Остальные десять компонент разбиваются на четыре группы, представители которых изображены на рис. 10. Эти четыре группы компонент определяют типы поведения кластеров в течение перестройки, показанные на рис. 6-9 соответственно. Каждая группа разбита на два столбца, определяющие соответственно вторые и третьи типы перестроек на указанных рисунках.

\section{§ 3. Обобщения для произвольных выпуклых гамильтонианов}

Субдифференциал. Все линейные формы, оценивающие производную вогнутой функции сверху, образуют выпуклое компактное множество

$$
\mathscr{D} f(x)=\left\{p \in \Lambda^{*} \mid f_{x}^{\prime}(v) \leqslant p \cdot v \quad \forall v \in \Lambda\right\},
$$

которое называется субдифберенциалом вогнутой функции $f$ в точке $x$. (Здесь $p \cdot v$ - это значение линейной формы на векторе.) Минимум значений субдифференциала вогнутой функции на данном векторе равен значению ее производной

$$
f_{x}^{\prime}(v)=\min _{p \in \mathscr{D} f(x)} p \cdot v
$$

ОПРЕДЕЛЕНИЕ $h$-ГРАДИЕНТА. Пусть $\Lambda$ - конечномерное вещественное линейное пространство. Назовем гамильтонианом $h: \Lambda^{*} \rightarrow \mathbb{R}$ произвольную гладкую выпуклую функцию на двойственном пространстве. Любая линейная 


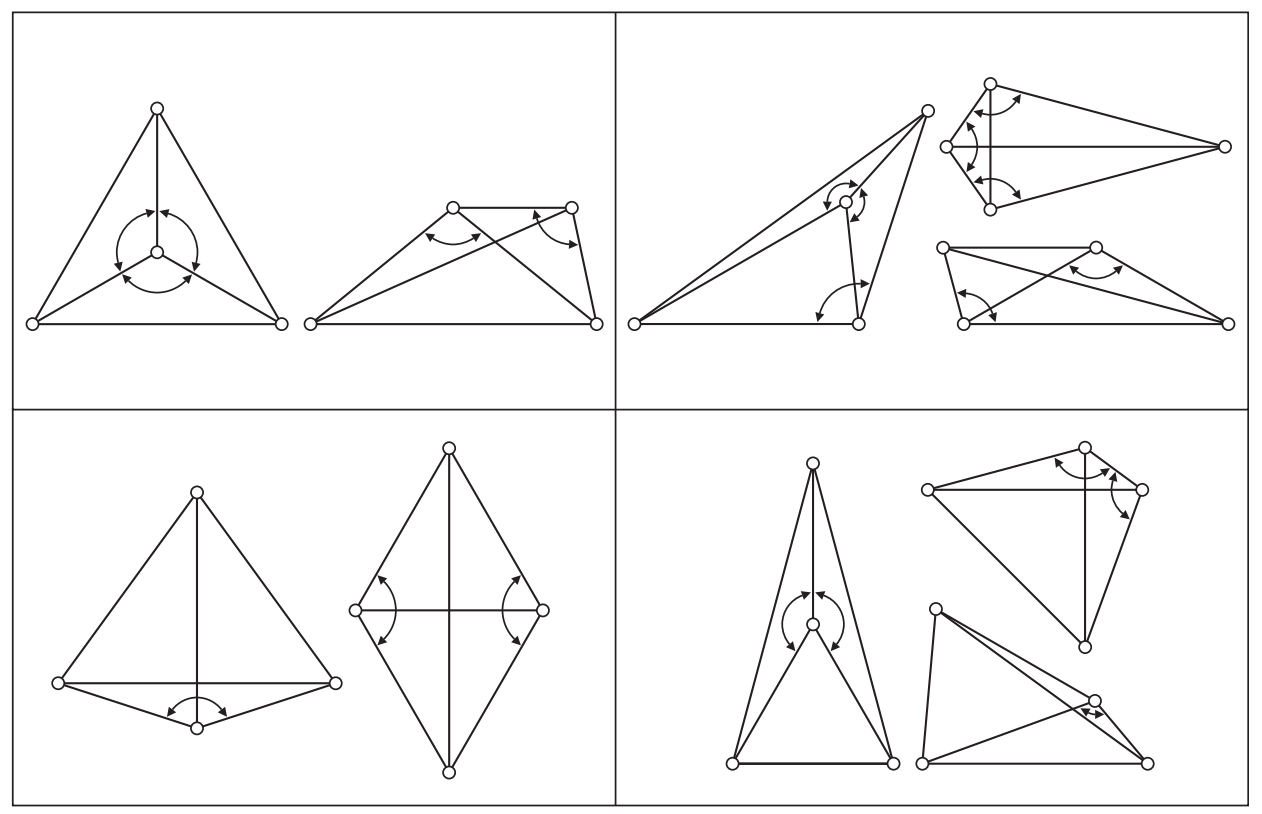

Рис. 11. Типичные наборы скоростей, определяющие рождение кластеров, остановку их роста, столкновение друг с другом и взаимодействие с нейтральными узлами

форма $d_{h} \varphi(\lambda) \in \mathscr{D} \varphi(\lambda)$, на которой гамильтониан $h$ достигает своего минимального значения на субдифференциале $\mathscr{D} \varphi(\lambda) \subset \Lambda^{*}$ относительно вогнутой функции $\varphi: \Lambda \rightarrow \mathbb{R}$, называется $h$-дифференииалом последней в точке $\lambda$. Хотя $h$-дифференциал не всегда определен однозначно, производная гамильтониана в нем

$$
\nabla_{h} \varphi(\lambda)=\left.\partial_{\varkappa} h\right|_{\varkappa=d_{h} \varphi(\lambda)} \in \Lambda, \quad \varkappa \in \Lambda^{*},
$$

не зависит от его выбора и называется $h$-градиентом относительно вогнутой функции $\varphi$ в точке $\lambda$.

Основное свойство $h$-градиента. Производная относительно вогнутой функции оценивается сверху ее $h$-дифференциалом, а на ее $h$-градиенте значения производной и $h$-дифференциала совпадают:

$$
\varphi_{\lambda}^{\prime}(v) \leqslant d_{h} \varphi(\lambda) \cdot v, \quad \varphi_{\lambda}^{\prime}\left(\nabla_{h} \varphi(\lambda)\right)=d_{h} \varphi(\lambda) \cdot \nabla_{h} \varphi(\lambda) .
$$

В самом деле, неравенство очевидно, поскольку по своему определению $h$-дифференциал принадлежит субдифференциалу. Кроме того,

$$
\varphi_{\lambda}^{\prime}\left(\nabla_{h} \varphi(\lambda)\right)=\min _{\varkappa \in \mathscr{D} \varphi(\lambda)} \varkappa \cdot \nabla_{h} \varphi(\lambda)=d_{h} \varphi(\lambda) \cdot \nabla_{h} \varphi(\lambda),
$$

поскольку $d_{h} \varphi(\lambda)$ - точка минимума гладкой функции $h$ на выпуклом множестве $\mathscr{D} \varphi(\lambda)$, и поэтому значение ее производной $\nabla_{h} \varphi(\lambda)$ на ковекторе $\varkappa-d_{h} \varphi(\lambda)$ неотрицательно для любого $\varkappa \in \mathscr{D} \varphi(\lambda)$. 
Лагранжева форма определения $h$-градиента. Лагранжиан - это преобразование Лежандра от гамильтониана:

$$
l: \Lambda \rightarrow \mathbb{R} \cup\{+\infty\}, \quad l(v)=\max _{\varkappa}\{\varkappa \cdot v-h(\varkappa)\}, \quad v \in \Lambda .
$$

По определению для любых $\varkappa \in \Lambda^{*}$ и $v \in \Lambda$

$$
h(\varkappa)+l(v) \geqslant \varkappa \cdot v,
$$

а для $h$-дифференциала и $h$-градиента относительно вогнутой функции достигается равенство, поскольку

$$
\left.\partial_{\varkappa}\{\varkappa \cdot v-h(\varkappa)\}\right|_{\varkappa=d_{h} \varphi(\lambda), v=\nabla_{h} \varphi(\lambda)}=0 .
$$

С учетом (8) это равенство переписывается в виде

$$
h\left(d_{h} \varphi(\lambda)\right)+l\left(\nabla_{h} \varphi(\lambda)\right)=d_{h} \varphi(\lambda) \cdot \nabla_{h} \varphi(\lambda)=\varphi_{\lambda}^{\prime}\left(\nabla_{h} \varphi(\lambda)\right) .
$$

Лемма 7. В данной точке $\lambda$ h-градиент минимизирует по $v$ величину

$$
l(v)-\varphi_{\lambda}^{\prime}(v)
$$

m.е. при $v=\nabla_{h} \varphi(\lambda)$ и фиксированном $\lambda$ указанная величина достигает своего минимума.

ДокАЗАТЕЛЬСтво. Согласно (8) и (9)

$$
\varphi_{\lambda}^{\prime}(v)-l(v) \leqslant d_{h} \varphi(\lambda) \cdot v-l(v) \leqslant h\left(d_{h} \varphi(\lambda)\right)=\varphi_{\lambda}^{\prime}\left(\nabla_{h} \varphi(\lambda)\right)-l\left(\nabla_{h} \varphi(\lambda)\right) .
$$

Связь с градиентом и полным градиентом. Пусть $\Lambda=E$ - конечномерное евклидово пространство с гамильтонианом $h(p)=|p|^{2} / 2$, где $p \in E^{*}$. Согласно лагранжеву определению $h$-градиент - это тот самый градиент, теория которого была построена в первой части настоящей статьи. Согласно же гамильтонову определению $h$-градиент относительно вогнутой функции - это вектор, сопряженный элементу ее субдифференциала минимальной длины. Это еще одно возможное определение градиента относительно вогнутой функции.

Если же $\Lambda=E \times \mathbb{R}$ - пространство-время с гамильтонианом

$$
h(p, q)=\frac{|p|^{2}}{2}+q, \quad p \in E^{*}, \quad q \in \mathbb{R}^{*},
$$

то

$$
l(v, s)=\left\{\begin{array}{ll}
\frac{|v|^{2}}{2} & \text { при } s=1, \\
+\infty & \text { при } s \neq 1,
\end{array} \quad v \in E, \quad s \in \mathbb{R},\right.
$$

и согласно лагранжеву определению пространственная компонента $h$-градиента - это полный градиент:

$$
\nabla_{h} \varphi(x, t)=(\operatorname{Grad} \varphi(x, t), 1)
$$


Согласно же гамильтонову определению пространственные компоненты $h$-дифференциала и $h$-градиента сопряжены друг другу. Поэтому

$$
d_{h} \varphi(x, t)(v, 0)=\langle\operatorname{Grad} \varphi(x, t), v\rangle \quad \forall v \in E,
$$

что вместе с неравенством (8) дает

$$
\varphi_{x, t}^{\prime}(v, 0) \leqslant\langle\operatorname{Grad} \varphi(x, t), v\rangle \quad \forall v \in E .
$$

Градиент решения произвольной вариационной задачи. Пусть $\varphi-$ не произвольная функция, а решение вариационной задачи в пространстве-времени $\Lambda=E \times \mathbb{R}$ с выпуклым лагранжианом $L: E \rightarrow \mathbb{R}, H: E^{*} \rightarrow \mathbb{R}$ - его преобразование Лежандра; а $h(p, q)=H(p)+q$, где $p \in E^{*}$ и $q \in \mathbb{R}^{*}$.

Тогда согласно А.Н. Соболевскому и К. М. Ханину пространственная компонента $h$-градиента находится из условия минимальности

$$
\max _{\left.p \in \mathscr{D} \varphi\right|_{t}(x)}\{H(p)-p \cdot v+L(v)\} \rightarrow \min
$$

относительно $v \in E$, где $\left.\varphi\right|_{t}(\cdot)=\varphi(\cdot, t)$ - решение вариационной задачи, рассматриваемое как функция только от пространственных переменных при фиксированном времени $t$. В частности, если $L(v)=|v|^{2} / 2$, то $H(p)=|p|^{2} / 2$ и пространственная компонента $h$-градиента $\nabla_{h} \varphi(x, t)$ сопряжена центру шара минимально возможного радиуса, который содержит субдифференциал решения $\varphi$, рассматриваемого как функция только от пространственных переменных при фиксированном времени.

Решение $h$-градиентного дифференциального уравнения с однородным потенциалом. Нижеследующая теорема 3 обобщает лемму 2 о существовании решения градиентного дифференциального уравнения с однородным потенциалом.

Теорема 3. Пусть $\varphi: \Lambda \rightarrow \mathbb{R}$ - вогнутая функиия, однородная степени 1:

$$
\varphi(c \lambda)=c \varphi(\lambda) \quad \forall c \geqslant 0 .
$$

Тогда кривая

$$
\xi(t)=t \nabla_{h} \varphi(0)
$$

является решением дифференциального уравнения

$$
\dot{\lambda}=\nabla_{h} \varphi(\lambda)
$$

с начальным условием $\xi(0)=0$ при всех $t \geqslant 0$.

ДокАЗАТЕЛЬСтво. Пусть $\lambda_{0}=\nabla_{h} \varphi(0)$ и $\varkappa_{0}=d_{h} \varphi(0)$. Нам нужно доказать, что $\nabla_{h} \varphi\left(t \lambda_{0}\right)=\lambda_{0}$ при всех $t \geqslant 0$. Это, в свою очередь, сразу следует из определения $h$-градиента и включений

$$
\varkappa_{0} \in \mathscr{D} \varphi\left(t \lambda_{0}\right) \subset \mathscr{D} \varphi(0)
$$


которые доказываются так. Согласно основному свойству $h$-градиента

$\varphi_{0}^{\prime}\left(t \lambda_{0}+v\right)-\varphi_{0}^{\prime}\left(t \lambda_{0}\right) \leqslant \varkappa_{0} \cdot\left(t \lambda_{0}+v\right)-t \varphi_{0}^{\prime}\left(\lambda_{0}\right)=\varkappa_{0} \cdot\left(t \lambda_{0}+v\right)-t \varkappa_{0} \cdot \lambda_{0}=\varkappa_{0} \cdot v$

при всех $v$. Но $\varphi=\varphi_{0}^{\prime}$ ввиду однородности. Поэтому

$$
\varphi\left(t \lambda_{0}+v\right)-\varphi\left(t \lambda_{0}\right) \leqslant \varkappa_{0} \cdot v \quad \forall v
$$

откуда в соответствии с определением производной вытекает, что

$$
\varphi_{t \lambda_{0}}^{\prime}(v) \leqslant \varkappa_{0} \cdot v \quad \forall v
$$

Но это и означает, что $\varkappa_{0} \in \mathscr{D} \varphi\left(t \lambda_{0}\right)$. Включение же $\mathscr{D} \varphi\left(t \lambda_{0}\right) \subset \mathscr{D} \varphi(0)$ вытекает из неравенства

$$
\varphi_{t \lambda_{0}}^{\prime}(v) \geqslant \varphi(v)=\varphi_{0}^{\prime}(v),
$$

которое следует из оценки (2).

Решение произвольного $h$-градиентного дифференциального уравнения. Сформулированная ниже гипотеза о существовании, единственности и непрерывной зависимости решения $h$-градиентного дифференциального уравнения остается недоказанной несмотря на доказанную выше теорему 3, обобщающую лемму 2 о существовании решения для однородного потенциала. Дело в том, что перенести лемму 3 о единственности и равномерно непрерывной зависимости решения на случай произвольного гамильтониана не удается.

ГиПотеЗА. Пусть $\Lambda$ - конечномерное вещественное линейное пространство, П $\subset \Lambda$ - его открытое выпуклое подмножество, $\Phi_{e, \Pi}-$ семейство всех функций на П, вогнутых относительно одной и той же гладкой функции $e: \Pi \rightarrow \mathbb{R}$, a $h: \Lambda^{*} \rightarrow \mathbb{R}-$ гладкий выпуклый гамильтониан. Тогда для любого

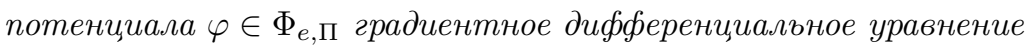

$$
\dot{\lambda}=\nabla_{h} \varphi(\lambda),
$$

где точка обозначает прочзводную по времени справа, при любом наперед заданном начальном условии

$$
\xi(0)=\lambda_{0} \in \Pi
$$

имеет непрерывное решение $\lambda=\xi(t)$ на некотором полуинтервале:

$$
\lim _{\Delta t \rightarrow+0} \frac{\xi(t+\Delta t)-\xi(t)}{\Delta t}=\nabla_{h} \varphi(\xi(t)) \quad \forall t \in[0, \delta), \quad \delta>0 .
$$

Такое решение единственно в том смысле, что любые два решения с одним и тем же начальным условием, но определенные на, быть может, разных полуинтервалах, совпадают на их пересечении. Кроме того, значение решения $\xi(t)$ непрерывно зависит от времени $t \geqslant 0$, начального условия $\lambda_{0}$ и потен-

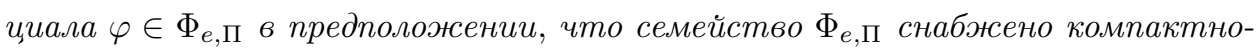
открытой топологией. 


\section{Список литературы}

[1] I. A. Bogaevsky, "Perestroikas of shocks and singularities of minimum functions", Physica D: Nonlinear Phenomena, 173:1-2 (2002), 1-28.

[2] Р. Рокафеллар, Выпукльй анализ, Мир, М., 1973.

[3] U. Frisch, J. Bec, "Burgulence", New trends in turbulence (Les Houches, 2000), eds. M. Lesieur, A. Yaglom, F. David, EDP Sci., Les Ulis, 2001, 341-383; arXiv: nlin. CD/0012033.

[4] С. Н. Гурбатов, А. Н. Малахов, А. И. Саичев, Нелинейные случайные волны в средах без дисперсии, Современные проблемы физики, 81, Наука, М., 1990.

[5] С. Н. Кружков, "Обобщенные решения нелинейных уравнений первого порядка со многими независимыми переменными. I", Матем. сб., 70(112):3 (1966), 394415.

[6] С. Н. Кружков, “Обобщенные решения нелинейных уравнений первого порядка со многими независимыми переменными. II", Матем. сб., 72(114):1 (1967), 108134.

[7] M. G. Crandall, L. C. Evans, P.-L. Lions, "Some properties of viscosity solutions of Hamilton-Jacobi equations", Trans. Amer. Math. Soc., 282:2 (1984), 487-502.

[8] L. C. Evans, P.E. Souganidis, "Differential games and representation formulas for solutions of Hamilton-Jacobi-Isaacs equations", Indiana Univ. Math. J., 33:5 (1984), 773-797.

И. А. Богаевский (I. A. Bogaevskiř)

Московский государственный университет

им. М. В. Ломоносова

E-mail: bogaevsk@mccme.ru
Поступила в редакцию

19.01.2006 